Article

\title{
Biomass Quantity and Quality from Different Year-Round Cereal-Legume Cropping Systems as Forage or Fodder for Livestock
}

\author{
Konapura Nagaraja Manoj ${ }^{1, *(D)}$, Bommalapura Gundanaik Shekara ${ }^{2}$, Shankarappa Sridhara ${ }^{3}$ (D), \\ Prakash Kumar Jha ${ }^{4}\left(\mathbb{D}\right.$ and P. V. Vara Prasad ${ }^{4,5}(\mathbb{D}$
}

Citation: Manoj, K.N.; Shekara, B.G.; Sridhara, S.; Jha, P.K.; Prasad, P.V.V. Biomass Quantity and Quality from Different Year-Round Cereal-Legume Cropping Systems as Forage or Fodder for Livestock. Sustainability 2021, 13, 9414. https://doi.org/ $10.3390 /$ su13169414

\section{Academic Editors: M.}

Iftikhar Hussain, Awais Shakoor, Sher Muhammad Shahzad, Taimoor Hassan Farooq and Marc A. Rosen

Received: 18 June 2021

Accepted: 18 August 2021

Published: 22 August 2021

Publisher's Note: MDPI stays neutral with regard to jurisdictional claims in published maps and institutional affiliations.

Copyright: (C) 2021 by the authors Licensee MDPI, Basel, Switzerland. This article is an open access article distributed under the terms and conditions of the Creative Commons Attribution (CC BY) license (https:// creativecommons.org/licenses/by/ $4.0 /)$.
1 Department of Agronomy, Gandhi Krishi Vignan Kendra, University of Agricultural Sciences, Bangalore 560 065, India

2 All India Coordinated Research Project (AICRP) on Forage Crops and Utilization, Zonal Agricultural Research Station, Vishweshwaraiah Canal Farm, Mandya 571 405, India; bgshekar66@gmail.com

3 Center for Climate Resilient Agriculture, University of Agricultural and Horticultural Sciences, Shivamogga 577 201, India; sridharas1968@gmail.com

4 Sustainable Intensification Innovation Lab, Kansas State University, Manhattan, KS 66506, USA; pjha@ksu.edu (P.K.J.); vara@ksu.edu (P.V.V.P.)

5 Department of Agronomy, Kansas State University, Manhattan, KS 66506, USA

* Correspondence: manojrajagri@gmail.com

\begin{abstract}
The quantity and quality of forage and fodder crops is the major drawback of the livestock sector in the country. There is a need to bridge the gap between the supply and demand of fodder through the adoption of specific sustainable fodder production strategies. The field experiments were conducted during kharif (rainy, June-October), rabi (post-rainy, October-February), and summer (March-May) seasons of 2018-19 and 2019-20 to identify a sustainable fodder cropping system module in randomized complete block design with fifteen fodder cropping systems in three replications. The main objective of this research was to identify the most productive cereal-legume cropping system, both in terms of quantity and quality of biomass, to reduce the gap between supply and demand of quality livestock feed around the year. Among cropping systems, Bajra-Napier hybrid intercropped with lucerne, cowpea, and sesbania recorded significantly higher green fodder (163.6, 155.2, and 144.0 t/ha/year, respectively) and dry matter yields (32.1, 30.8, and 31.3 t/ha/year, respectively). Similarly, the same perennial systems also recorded higher quality yield and ash content. However, higher crude protein content was noticed in monocrop legumes, with the highest in sesbania $(22.32 \%)$, while higher ether extractable fat was found in monocrop sesbania (3.78\%). The monocrop oats recorded higher non-fiber carbohydrates $(36.90 \%)$ while a monocrop of pearl millet recorded higher total carbohydrates $(80.75 \%)$, however they were on par with other monocrop cereal cropping systems. Cultivation of legumes as a monocrop, and their inclusion as an intercrop with cereals resulted in lower fiber fractions and improved crude protein in intercropping systems. Furthermore, this improved the dry matter intake and digestibility of fodder. With higher sustainable yield index values and land-use efficiency, perennial intercropping systems were also found to be sustainable. Thus, cultivation of the Bajra-Napier hybrid with either lucerne, cowpea, or sesbania as an intercrop will help livestock farmers to achieve higher productivity in terms of quantity and quality, and forms a viable option for overcoming livestock feed scarcity.
\end{abstract}

Keywords: fodder yield; cropping systems; quality; fiber fractions; sustainability

\section{Introduction}

Livestock are major component of the global agricultural production systems. The shift from calorie-based diets to protein rich diets of the burgeoning population gave impetus to grass-based livestock production in last few decades. Moreover, it offers a livelihood opportunity for the global smallholder farming community. In India, livestock 
production is mainly based upon mixed farming, in which farmers often integrate crops and livestock to maximize income and diversify the use of available resources. Livestock production is considered the backbone of Indian agriculture, as it contributes $7 \%$ to national gross domestic product (GDP) and 24.72\% to agricultural GDP [1].

According to the National Commission on Agriculture, the recommended area for animal feed (forage or fodder) production should be $10 \%$ of arable land i.e., 16.5 million hectares ( $\mathrm{m}$ ha). This being said, the area under animal feed cultivation has decreased due to increased cultivation of food and commercial crops [2]. At present, only $8.6 \mathrm{~m}$ ha area is devoted to livestock feed production in India. Furthermore, there is no standard practice of forage or fodder production in rural areas, and animals are fed with naturally grown grasses, shrubs, and weeds in the agricultural lands, which are of low quality in terms of protein, minerals, and available energy. It has been estimated that feed cost alone accounts for $60 \%$ of the milk production cost, as the livestock producers mainly rely on concentrate animal feeds for achieving higher milk yield [3]. Green forage or fodder that is rich in proteins, minerals, vitamins, carbohydrates, and micronutrients, as well as having a high dry matter intake, digestibility, and low fiber content, would help to lower the cost of livestock feeding [4] and increase animal productivity.

The above-mentioned challenges of the livestock sector are creating tremendous pressure on the country's available total feed and fodder resources. In this context, the cultivation of green fodder or forage crops which can produce greater quantity and quality of biomass will play an important role [5]. At present in India, the availability of green fodder, dry fodder and concentrates are 734.2, 326.4, and 61.0 million tonnes, respectively; this is against the current requirement of $827.2,426.1$, and 85.8 million tonnes, with a net deficit of $11.24 \%, 23.40 \%$, and $28.90 \%$, respectively [6]. Similarly, in Karnataka, the supply is 85,15 , and 7.5 million tonnes, respectively, compared to the current requirement of $122,25.4$, and 29.5 million tonnes, resulting in a net deficit of $30 \%, 40.95 \%$, and $74.50 \%$, respectively [7]. To meet the current forage and fodder demand of livestock population, the deficit must be met by either enhancing the productivity of existing animal feed crops or utilizing unconventional fodders and trees along with existing cropping systems. Moreover, increase in the acreage of livestock feeding crops, seems impossible due to increased land use competition by a burgeoning human population [8].

In this context, cereal-legume cropping systems involving annual and perennial fodder or forage crops with high yielding varieties and hybrids is a viable option to meet the forage and fodder demand. Sustainable intensification of livestock feed through increased productivity maintains continuous cover on the soil and has many other benefits besides supplying animal feed. These benefits include reduced incidence of pests and diseases, suppressing weed population, reduced erosion, and additional nitrogen supply through atmospheric nitrogen fixation $[9,10]$. Higher dry matter yields were realized with sorghum and lima bean intercropping systems at different planting proportions [11]. With maximum utilization of land area and other resources, pearl millet intercropped with cluster bean produced higher forage yield with improved forage quality [12]. The higher productivity of forage and fodder crop under cereal-legume intercropping systems has also been well established $[1,2,8,13]$.

Globally, cereal-legume cropping system-based forage cultivation mitigates the impact of greenhouse gases through reduced use of inorganic nitrogen application and enhancing soil carbon stocks [9], reducing runoff losses [10], drought resilience [13], and improving livelihood and profitability [5]. These types of intercropping are practiced more in countries with low inputs than the intensified high-input farming systems [9]. However, in last two decades, with growing interests in conservation agriculture and growing feed demand for intensive dairy-based farming, cereal-legume-based fodder cultivation has increased in developed countries. With the increasing awareness about resiliency and sustainability of farming system, the importance of these cropping system has been realized by associated stakeholders. 
However, very little information is available on the quality of green forage or fodder production in a year-round system of intercropping different annual and perennial crops and their seasonal rotations. Therefore, the main objective of this research is to identify the most productive cereal-legume cropping system, both in terms of quantity and quality of biomass, to reduce the gap between supply and demand of quality livestock feed around the year.

\section{Materials and Methods}

\subsection{Site Description and Weather}

Field experiments were conducted during the kharif (rainy season, June-October), rabi (post rainy, October-February) and summer (March-May) seasons of 2018-19 and 2019-20 at Zonal Agricultural Research Station, Vishweshwaraiah Canal Farm, Mandya, Karnataka, India. This falls under the southern dry zone (ACZ-VI) of Karnataka between $12^{\circ} 45^{\prime}$ and $13^{\circ} 57^{\prime} \mathrm{N}$ latitude and $76^{\circ} 45^{\prime}$ and $78^{\circ} 24^{\prime} \mathrm{E}$ longitude at an altitude of $695 \mathrm{~m}$ above mean sea level. The location belongs to dry tropical savanna climate as per Koppen's classification, which often experiences dry seasons with severe drought conditions (http: / / koeppen-geiger.vu-wien.ac.at; accessed on 8 August 2021). The soil at the experimental site was classified as "Typic Kandiustalf" as per United States Department of Agricultural classification. The soil of the site was red sandy loam in texture, with neutral $\mathrm{pH}(7.45)$, medium electrical conductivity $(0.38 \mathrm{ds} / \mathrm{m})$, and low organic carbon concentration $(0.55 \%)$, having a nutrient profile of $265.40 \mathrm{~kg} \mathrm{~N} / \mathrm{ha}, 49.25 \mathrm{~kg} \mathrm{P}_{2} \mathrm{O}_{5} / \mathrm{ha}$ and $162.35 \mathrm{~kg} \mathrm{~K} \mathrm{~K}_{2} \mathrm{O} / \mathrm{ha}$. The total amount of rainfall received during the cropping season was $520.7 \mathrm{~mm}$ during 2018-19 and $912.7 \mathrm{~mm}$ during 2019-20 (Figure 1). The year-round cropping period was from June to May during both the years of the experiment. The peak rainfall was received in September (177.8 mm) during 2018-19 and in October (331.2 mm) during 2019-20. However, during the dry period, lifesaving irrigation was provided to the crops once in fifteen days with water belonging to irritability class 2 as a protective measure. The maximum temperature was recorded in May $\left(35.5^{\circ} \mathrm{C}\right)$, and minimum in January $\left(17^{\circ} \mathrm{C}\right)$ during $2018-19$, whereas in 2019-20 the maximum temperature of $36^{\circ} \mathrm{C}$ was recorded in June and the minimum temperature of $16.3^{\circ} \mathrm{C}$ in December. Maximum relative humidity was observed in August (95\%), and minimum in May (53\%) during 2018-19, whereas in 2019-20 the maximum relative humidity of $92 \%$ was observed in October and the minimum of $35 \%$ in March (Figure 1a,b).

\subsection{Experimental Details}

The experiment was laid out in a Randomized Complete Block Design (RCBD), comprising of fifteen different forage or fodder cropping systems with three replications (Table 1). The investigation comprised of five cereal crops (maize (variety African Tall), sorghum (variety Sudex Chari-1), pearl millet (variety BAIF bajra-1), oats (variety OS-6), and the Bajra-Napier hybrid (variety BNH-10)) and four legume crops (Cowpea (variety MFC-09-1), Lucerne (variety RL-88), Desmanthus (variety Co-1), and Sesbania (variety Local)). Sowing was carried out using a manually operated marker by opening furrows to a depth of $3 \mathrm{~cm}$. Seeds of main and intercrops were line sown at recommended row proportion as per the treatments at spacing of $30 \mathrm{~cm} \times 10 \mathrm{~cm}$. In Bajra-Napier hybrid-based cropping systems, two eye budded well-matured Napier stem cuttings were planted in paired row system with an inter-row spacing of $60 \mathrm{~cm}$ and intra-row spacing of $45 \mathrm{~cm}$. Intercrops were sown as a replacement series between the two paired rows. The perennial crops were sown only once at the initial time of the experiment, while sowing of annual crops was taken up in each season accordingly. The crop calendar for annual and perennials is shown in Figure 2a,b for both the years (2018-2019, 2019-2020). As perennial cropping systems remained in the field around the year, we have taken all the crops included in the experiment in all three seasons as monocrops for better comparison of the systems. Chemical fertilizers were applied in furrows and mixed properly at the time of sowing as per the package of practices. In annual crops, $50 \%$ of the recommended nitrogen $(\mathrm{N})$ was 
applied at the time of sowing, and the remaining $\mathrm{N}$ was top-dressed at 30 days after sowing, while in perennial crops $10 \%$ of $\mathrm{N}$ was applied as basal dose, and the remaining $90 \%$ was applied in two equal splits after each cut. A full dose of recommended phosphorus (P) and potassium $(\mathrm{K})$ were applied at the time of sowing.
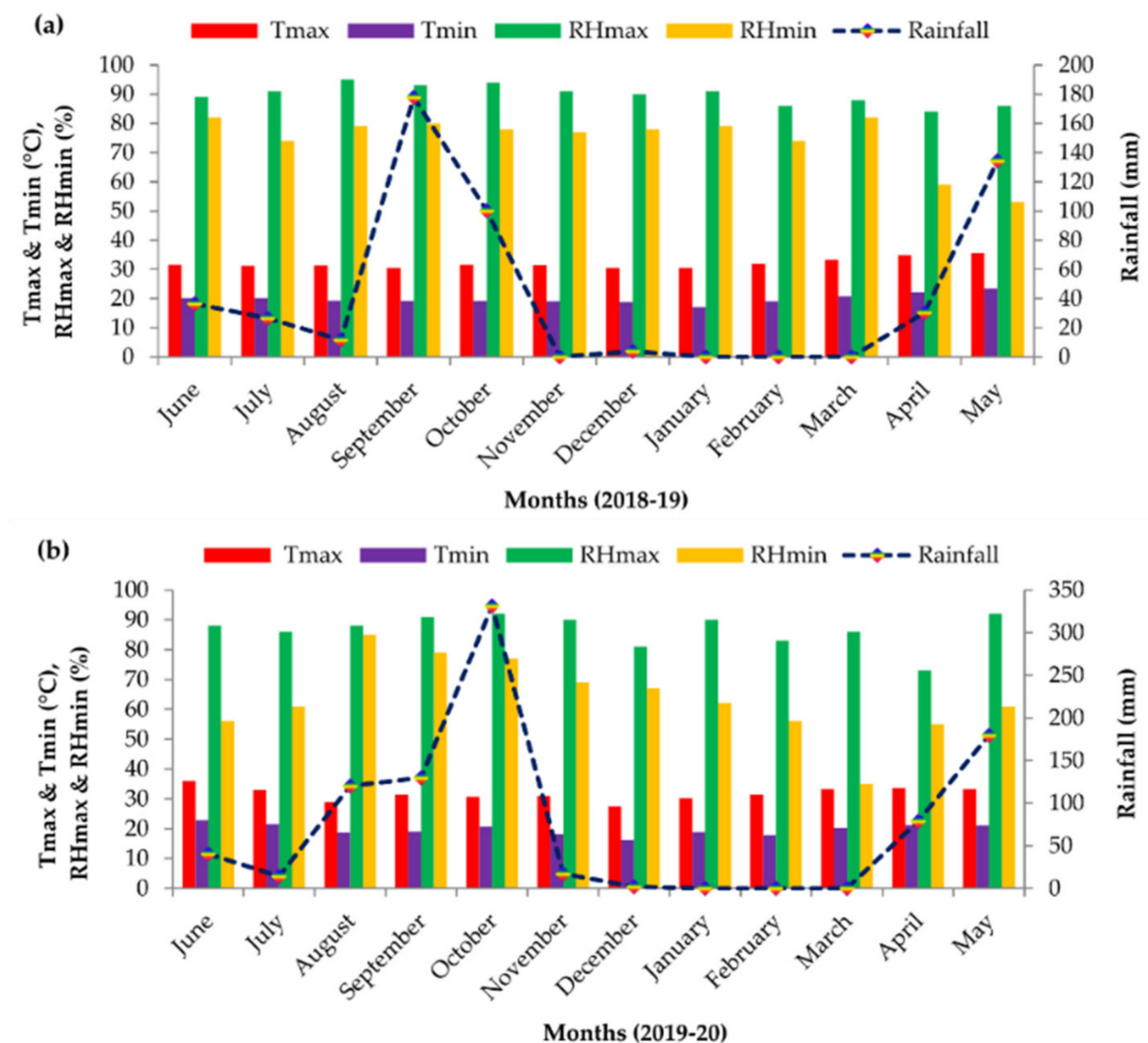

Figure 1. Weather conditions (minimum (min) and maximum (max) temperatures $(\mathrm{T})$, relative humidity (RH), and monthly rainfall) during the two experimental years: 2018-19 (a) and 2019-20 (b) at the research site (Zonal Agricultural Research Station, Mandya, Karnataka, India).

Table 1. Treatment details of different cropping systems implemented at the experimental site.

\begin{tabular}{|c|c|c|}
\hline T.No. & Treatment Details & Abbreviations \\
\hline 1 & $\begin{array}{l}\text { Fodder Maize-Fodder Maize-Fodder Maize } \\
\text { monocropping system }\end{array}$ & Mono: MZ-MZ-MZ \\
\hline 2 & $\begin{array}{l}\text { Fodder Sorghum-Fodder Sorghum-Fodder } \\
\text { Sorghum monocropping system }\end{array}$ & Mono: SG-SG-SG \\
\hline 3 & $\begin{array}{l}\text { Fodder Oats-Fodder Oats-Fodder Oats } \\
\text { monocropping system }\end{array}$ & Mono: OT-OT-OT \\
\hline 4 & $\begin{array}{l}\text { Fodder Pearl millet-Fodder Pearl millet-Fodder } \\
\text { Pearl millet monocropping system }\end{array}$ & Mono: PM-PM-PM \\
\hline 5 & $\begin{array}{l}\text { Fodder Cowpea-Fodder Cowpea-Fodder } \\
\text { Cowpea monocropping system }\end{array}$ & Mono: $\mathrm{CP}-\mathrm{CP}-\mathrm{CP}$ \\
\hline 6 & Bajra-Napier hybrid perennial system & Perennial: BNH \\
\hline 7 & Lucerne perennial system & Perennial: LU \\
\hline 8 & Desmanthus perennial system & Perennial: DS \\
\hline 9 & Sesbania perennial system & Perennial: SE \\
\hline 10 & $\begin{array}{c}\text { Fodder Maize }+ \text { Cowpea (3:1)-Fodder Oat }+ \\
\text { Cowpea (3:1)-Pearl millet }+ \text { Cowpea (3:1)-Mixed } \\
\text { cropping }\end{array}$ & $\begin{array}{l}\text { Mixed: } \mathrm{MZ}+\mathrm{CP}-\mathrm{OT}+ \\
\quad \mathrm{CP}-\mathrm{PM}+\mathrm{CP}\end{array}$ \\
\hline
\end{tabular}


Table 1. Cont.

\begin{tabular}{|c|c|c|}
\hline T.No. & Treatment Details & Abbreviations \\
\hline 11 & $\begin{array}{l}\text { Fodder Sorghum + Cowpea (3:1)-Fodder Maize } \\
+ \text { + Cowpea }(3: 1)-\text {-Pearl millet + Cowpea }(3: 1)\end{array}$ & $\begin{array}{l}\text { Mixed: SG + CP - MZ + } \\
\text { CP-PM + CP }\end{array}$ \\
\hline 12 & $\begin{array}{l}\text { Bajra-Napier hybrid + Cowpea (2:8) perennial } \\
\text { system }\end{array}$ & Perennial: $\mathrm{BNH}+\mathrm{CP}$ \\
\hline 13 & $\begin{array}{l}\text { Bajra-Napier hybrid + Lucerne (2:8) perennial } \\
\text { system }\end{array}$ & Perennial: BNH + LU \\
\hline 14 & $\begin{array}{c}\text { Bajra-Napier hybrid + Desmanthus (2:8) } \\
\text { perennial system }\end{array}$ & Perennial: BNH + DS \\
\hline 15 & $\begin{array}{c}\text { Bajra-Napier hybrid + Sesbania (2:8) perennial } \\
\text { system }\end{array}$ & Perennial: BNH + SE \\
\hline
\end{tabular}

\subsection{Biomass Measurements}

The crops were harvested as per the schedule based on crop developmental stage. The seasonal maize and sorghum were harvested at milking and full flowering stage, while pearl millet, oats, and cowpea were harvested at 50\% flowering stage. For perennials, Bajra-Napier hybrid, lucerne, desmanthus, and sesbania, the first harvest was taken at $70,60,90$, and 180 days after sowing, respectively, by leaving stubbles of suitable height. Subsequent harvests were taken at 35-45 days, 25-30 days, 45-50 days, and 45-50 days intervals, respectively, based on the crop growth and developmental stages. Crops from the net area of each experimental plot were harvested separately, weighed in kilograms with the help of a spring balance at each cut, converted to the hectare, and expressed as the green fodder yield (GFY) in quintals per hectare. Later, dry matter (DM) content was determined by drying a known quantity of representative fresh samples from each plot immediately after harvest, and samples were oven-dried at $70 \pm 2{ }^{\circ} \mathrm{C}$ for $48 \mathrm{~h}$ and weighed. After that, dry matter content was determined, and dry matter yield (DMY) was calculated by multiplying the DM content with GFY.

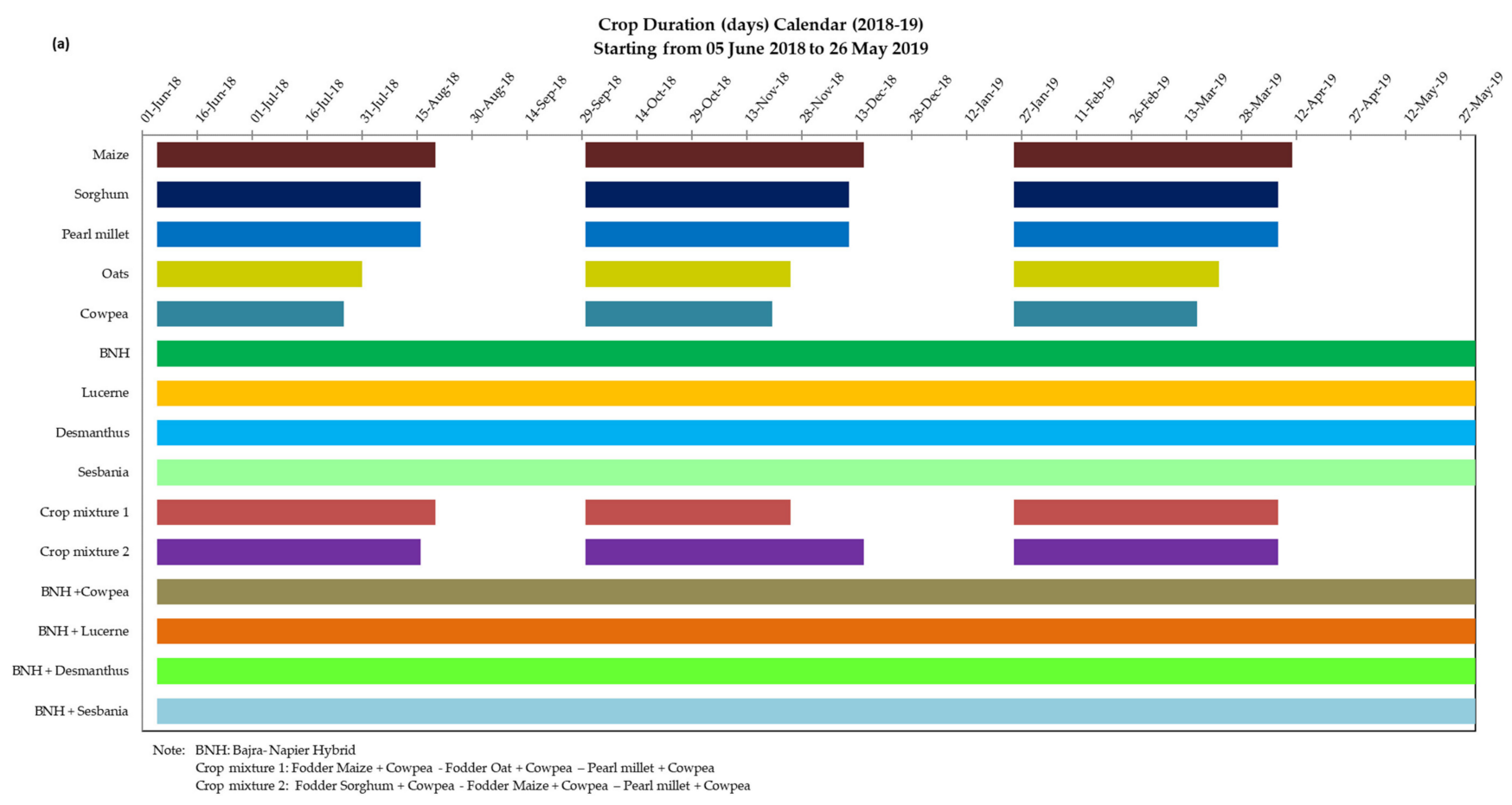

Figure 2. Cont. 
(b)

Crop Duration (days) Calender (2019-20)

Starting from 27 May 2019 to 24 May 2020

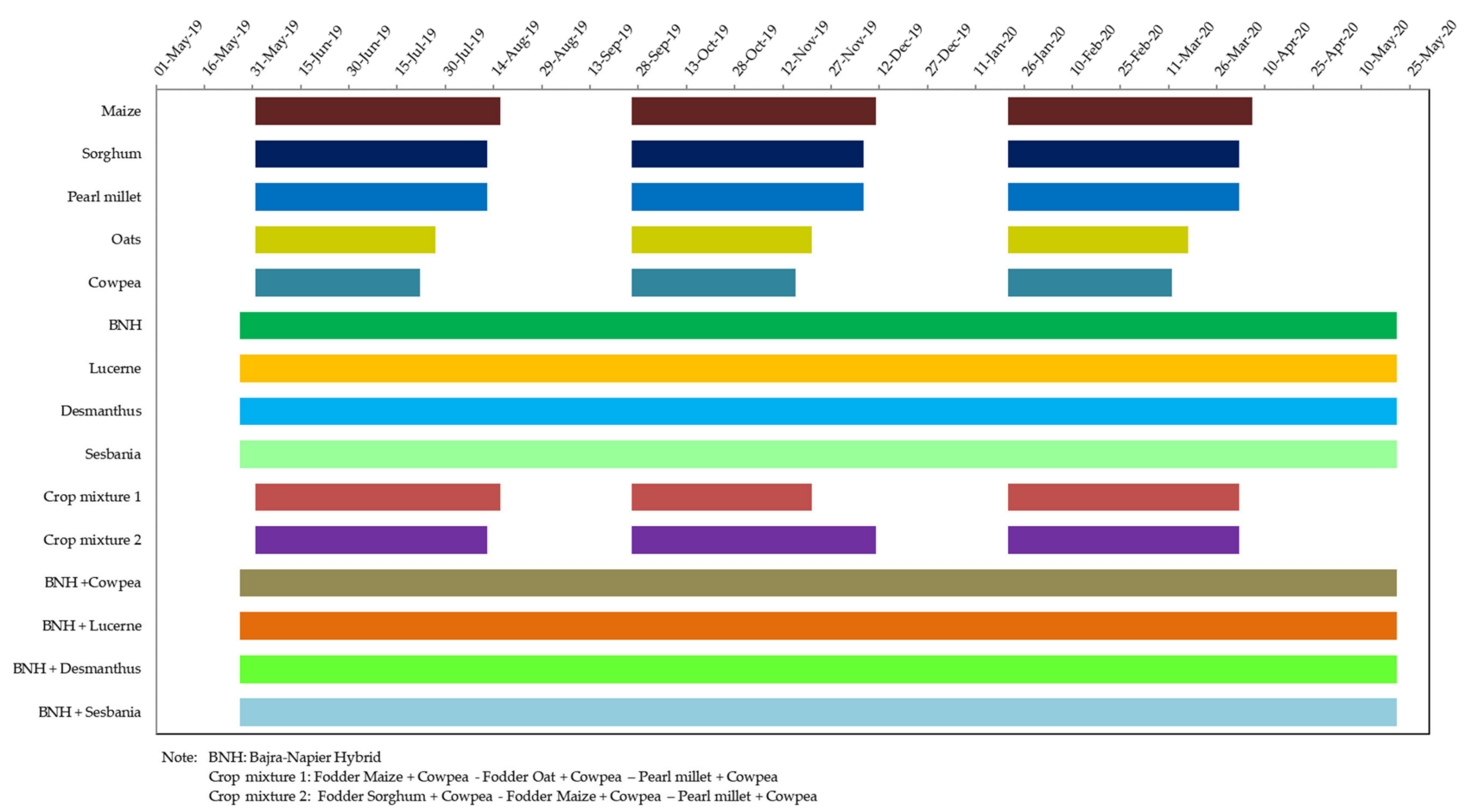

Figure 2. Crop sowing and harvest schedule for annuals and perennials in 2018-2019 (a) and 2019-2020 (b).

\subsection{Quality Parameters and Fiber Fractions}

After harvest, plant samples from each treatment were collected, oven-dried, powdered, and used to analyze quality parameters. In the intercropping systems, the plant samples from the main and intercrops were mixed as per the adopted row proportion, and further used for the analysis. The dried samples were ground in Willey mill using a $2 \mathrm{~mm}$ sieve for estimation of quality parameters and a $1 \mathrm{~mm}$ sieve for fiber fractions analysis. The fiber fractions included neutral detergent fiber (NDF) and acid detergent fiber (ADF). The NDF represents the total fraction of fiber (cellulose, hemicellulose, and lignin) which makes up the structural component (carbohydrates or sugars) of the cell wall. Conversely, $\mathrm{ADF}$ is the least digestible plant component which includes cellulose and lignin. The ADF values are inversely related to digestibility. Crude fat percent in the biomass was estimated using ether extractable method. The quality parameters were estimated according to the standard procedure recommended by AOAC [14] protocol, and their respective yields were calculated by multiplying with the dry matter yield of the crops. All the forage quality parameters are reported on a dry matter (DM) basis, or per unit weight of DM. However, non-fiber carbohydrate $(\mathrm{CHO})$, total $\mathrm{CHO}$ percent, and total digestible crude protein yield (TDCPY) was determined according to the following equations [15].

$$
\begin{gathered}
\text { Non-fiber } \mathrm{CHO}(\%)=100-(\text { crude protein }+ \text { fat }+ \text { fiber }+ \text { ash }+ \text { moisture }) \\
\text { Total CHO }(\%)=100-(\text { crude protein }+ \text { fat }+ \text { ash }) \\
\text { TDCPY }(\%)=[0.97 \times \text { crude protein yield }]-0.67
\end{gathered}
$$

Dry matter intake (DMI), digestible dry matter (DDM) and relative feed value (RFV) were calculated according to the following formula adopted from Horrocks and Vallentine [16]:

DMI $(\%$ body weight $)=120 / \mathrm{NDF}(\%)$ on dry matter basis

$\operatorname{DDM}(\%)=88.9-(0.779 \times \%$ ADF on dry matter basis $)$ 


$$
\mathrm{RFV}(\%)=\% \mathrm{DDM} \times \% \mathrm{DMI} \times 0.775
$$

\subsection{Land Use Efficiency and Sustainable Yield Index}

Land use efficiency (LUE) and sustainable yield index (SYI) were calculated for each cropping system module to assess efficiency and sustainability. LUE is the total number of days a field remains occupied by the crops in a cropping system during an agriculture year [17]. A higher LUE value denotes efficient utilization of land in a year.

$$
\mathrm{LUE}=\frac{\sum_{i=1}^{n} D i}{365} \times 100
$$

where $i=0,1,2,3 \ldots, n ; n=$ total number of crops; and $D i=$ number of days occupied by ith crop.

The trend of yield over a year in a system reflects the sustainable yield of a cropping system. SYI values range from 0 to 1 . The value nearing unity shows higher stability reflecting that the system is more sustainable [18].

$$
\mathrm{SYI}=\frac{\mathrm{Y}-\mathrm{SD}}{\mathrm{Y}_{\max }}
$$

where $\mathrm{Y}=$ average yield of the management practice over the years $(n=0,1,2,3 \ldots)$; $\mathrm{SD}=$ standard deviation of the yield of the management practice over years; and $\mathrm{Y}_{\max }=$ observed maximum yield of the experiment.

\subsection{Statistical Analysis}

The experimental data collected on various parameters were subjected to statistical analysis adopting Fisher's method of analysis of variance (ANOVA) outlined by Gomez and Gomez [19]. The least significant difference (LSD) was used to compare treatment means at $5 \%$ level of significance $(p<0.05)$. In the case of significant results, the critical difference at $5 \%$ level of probability was calculated to test the difference between two treatment means. Correlation graphs were plotted using R software. Initially, analysis was performed separately for both the years of study, and a similar kind of response was observed. Hence, all the results in the present study are interpreted on the pooled mean basis of two years' data.

\section{Results}

\subsection{Biomass Yield and Dry Matter Concentration}

The cropping systems involving cereal-legume perennial intercropping achieved significantly higher green fodder yield (GFY) and dry matter yield (DMY) compared to cereal and legume monocrop throughout the year (Table 2). Bajra-Napier hybrid produced significantly higher GFY and DMY than other cropping systems, when intercropped at 2:8 row proportion with legumes such as lucerne (163.6 and 32.1 t/ha/year, respectively), cowpea (155.2 and $30.8 \mathrm{t} /$ ha/year, respectively) and sesbania (144.0 and $31.3 \mathrm{t} / \mathrm{ha}$ /year, respectively). However, when cowpea, desmanthus, oats, and pearl millet cultivated as monocrops throughout the year resulted in significantly lower GFY, and the magnitude of decrease was almost $110 \%, 106 \%, 98 \%$, and $92 \%$, respectively, as compared to the superior Bajra-Napier hybrid + lucerne system which had better GFY. Similarly, the same monocrops had significantly lower DMY, and account for only $48 \%, 49 \%, 52 \%$, and $56 \%$ DMY, respectively, compared to that achieved by the Bajra-Napier hybrid + lucerne perennial system. 
Table 2. Green fodder yield (GFY), dry matter yield (DMY), and dry matter concentration (DM) as influenced by different cropping system treatments.

\begin{tabular}{|c|c|c|c|c|}
\hline T. No. & Abbreviations & GFY (t/ha/Year) & DMY (t/ha/Year) & DM (\%) \\
\hline 1 & Mono: MZ-MZ-MZ & $104.7 \pm 5.544^{\text {fgh }}$ & $21.5 \pm 0.731^{\text {cdef }}$ & $20.52 \pm 0.607^{b c d}$ \\
\hline 2 & Mono: SG-SG-SG & $90.6 \pm 5.526^{\mathrm{hi}}$ & $20.8 \pm 0.285$ def & $22.99 \pm 1.672^{a}$ \\
\hline 3 & Mono: OT-OT-OT & $82.7 \pm 5.854^{i}$ & $16.7 \pm 1.328^{g}$ & $20.20 \pm 0.635^{\mathrm{bcd}}$ \\
\hline 4 & Mono: PM-PM-PM & $85.4 \pm 3.659^{\mathrm{i}}$ & $17.8 \pm 1.298^{\mathrm{fg}}$ & $20.89 \pm 0.318^{b c d}$ \\
\hline 5 & Mono: $\mathrm{CP}-\mathrm{CP}-\mathrm{CP}$ & $77.8 \pm 13.325^{\mathrm{i}}$ & $15.5 \pm 1.317^{g}$ & $19.98 \pm 0.087^{c d}$ \\
\hline 6 & Perennial: BNH & $126.2 \pm 1.271^{\text {cde }}$ & $24.8 \pm 0.088^{b c}$ & $19.62 \pm 0.742^{\mathrm{d}}$ \\
\hline 7 & Perennial: LU & $95.2 \pm 0.578$ ghi & $19.1 \pm 1.828^{\text {efg }}$ & $20.02 \pm 0.642^{\mathrm{cd}}$ \\
\hline 8 & Perennial: DS & $79.6 \pm 0.617^{i}$ & $15.7 \pm 1.271 \mathrm{~g}$ & $19.70 \pm 1.288^{\mathrm{cd}}$ \\
\hline 9 & Perennial: SE & $96.0 \pm 9.416$ ghi & $21.2 \pm 0.433^{\text {cdef }}$ & $22.10 \pm 0.634^{\mathrm{ab}}$ \\
\hline 10 & $\begin{array}{c}\text { Mixed: } \mathrm{MZ}+\mathrm{CP}-\mathrm{OT}+ \\
\mathrm{CP}-\mathrm{PM}+\mathrm{CP}\end{array}$ & $110.3 \pm 2.696^{\text {efg }}$ & $22.7 \pm 1.595$ bcde & $20.59 \pm 0.994^{\mathrm{bcd}}$ \\
\hline 11 & $\begin{array}{c}\text { Mixed: SG + CP-MZ + } \\
\text { CP-PM + CP }\end{array}$ & $115.4 \pm 5.868$ def & $24.4 \pm 1.074^{\mathrm{bcd}}$ & $21.20 \pm 0.692^{\mathrm{abcd}}$ \\
\hline 12 & Perennial: $\mathrm{BNH}+\mathrm{CP}$ & $155.2 \pm 13.279 \mathrm{ab}$ & $30.8 \pm 0.145^{\mathrm{a}}$ & $19.81 \pm 0.754^{\mathrm{cd}}$ \\
\hline 13 & Perennial: BNH + LU & $163.6 \pm 1.037^{\mathrm{a}}$ & $32.1 \pm 0.291^{\mathrm{a}}$ & $19.58 \pm 1.102^{d}$ \\
\hline 14 & Perennial: BNH + DS & $131.0 \pm 6.970^{\mathrm{cd}}$ & $25.5 \pm 2.773^{b}$ & $19.48 \pm 0.297^{\mathrm{d}}$ \\
\hline 15 & $\begin{array}{c}\text { Perennial: BNH + SE } \\
\text { SEM }\end{array}$ & $\begin{array}{c}144.0 \pm 4.020 \mathrm{bc} \\
6.3\end{array}$ & $\begin{array}{c}31.3 \pm 2.419^{\mathrm{a}} \\
1.3\end{array}$ & $\begin{array}{c}21.72 \pm 0.789 \mathrm{abc} \\
0.70\end{array}$ \\
\hline & $\operatorname{LSD}(p=0.05)$ & 18.3 & 3.8 & 2.03 \\
\hline
\end{tabular}

SEM: Standard Error of Means; LSD: Least Significant Difference. Superscript represents treatment means with similar alphabets do not differ significantly at LSD $=0.05$ probability $(p)$ level. Note: MZ, Maize; SG, Sorghum; OT, Oats; PM, Pearl millet; CP, Cowpea; BNH, Bajra Napier Hybrid; LU, Lucerne; DS, Desmanthus; SE, Sesbania.

In the present study, the DM concentration of fodder was significantly influenced by different cropping system modules (Table 2). The monocropping system of sorghum $(22.99 \%)$, sesbania $(22.10 \%)$, the perennial system of Bajra-Napier hybrid + sesbania $(21.72 \%)$, and the crop mixture of fodder sorghum + cowpea-fodder maize + cowpeapearl millet + cowpea $(21.20 \%)$ has shown statistically higher dry matter concentration than other cropping systems. Significantly lower DM concentration was observed in monocrop Bajra-Napier hybrid fodder biomass. Furthermore, intercropping of legumes viz., cowpea, lucerne, and desmanthus with Bajra-Napier hybrid has shown a reduced DM content in their fodder when compared to their sole crops.

\subsection{Quality Traits}

The nitrogen and protein in fodder is often considered a good determinant of forage quality. Cropping systems involving legumes showed significantly higher nitrogen $(\mathrm{N})$ and crude protein $(\mathrm{CP})$ compared to cereals cultivated throughout the year (Table 3). Compared to other cropping systems, significantly higher $\mathrm{N}$ and $\mathrm{CP}$ concentrations were found in monocultures of legumes such as sesbania ( $3.57 \%$ and $22.32 \%$, respectively), lucerne (3.34\% and $20.62 \%$, respectively), and cowpea (3.28\% and $20.51 \%$, respectively). The cereals grown as monocrops have recorded lower $\mathrm{N}$ and $\mathrm{CP}$ concentration, but they have shown a significant improvement in these concentrations when they are intercropped with legume fodder crops. On the other hand, ash concentration indicates the mineral matter composition of fodder and is significantly higher in perennial Bajra-Napier hybrid + cowpea (11.22\%) and Bajra-Napier hybrid + sesbania (11.18\%) systems fodder. However, these systems were found statistically on par with monocropping systems viz., sesbania, lucerne, Bajra-Napier hybrid, and perennial Bajra-Napier hybrid + lucerne systems. In contrast, pure culture of pearl millet $(8.12 \%)$, sorghum $(8.26 \%)$, and oats $(8.44 \%)$ throughout the year has shown significantly lower ash concentration among all the fodder cropping systems (Table 3). 
Table 3. Nitrogen $(\mathrm{N})$, crude protein $(\mathrm{CP})$, ash, fat, non-fiber $\mathrm{CHO}(\mathrm{NF}-\mathrm{CHO})$, and total carbohydrate (T-CHO) percent in biomass as influenced by different cropping system treatments.

\begin{tabular}{|c|c|c|c|c|c|c|c|}
\hline T. No. & Abbreviations & N (\%) & CP (\%) & Ash (\%) & Fat $(\%)$ & NF-CHO (\%) & T-CHO (\%) \\
\hline 1 & Mono: MZ-MZ-MZ & $1.58 \pm 0.023 \mathrm{ghi}$ & $9.92 \pm 0.367^{\mathrm{fgh}}$ & $10.18 \pm 0.268^{\text {bcde }}$ & $2.67 \pm 0.025 \mathrm{gh}$ & $32.84 \pm 1.209^{b c}$ & $77.23 \pm 1.995^{\mathrm{abc}}$ \\
\hline 2 & Mono: SG-SG-SG & $1.30 \pm 0.003^{\mathrm{i}}$ & $8.10 \pm 0.5^{h}$ & $8.26 \pm 0.273 \mathrm{gh}$ & $3.09 \pm 0.052^{\mathrm{bcd}}$ & $34.19 \pm 1.109^{a b}$ & $80.55 \pm 0.041^{a}$ \\
\hline 3 & Mono: OT-OT-OT & $1.66 \pm 0.009^{\mathrm{fgh}}$ & $10.36 \pm 0.26^{\text {efg }}$ & $8.44 \pm 0.092$ gh & $3.03 \pm 0.156^{\text {bcde }}$ & $36.9 \pm 3.582^{a}$ & $78.16 \pm 1.659^{a b}$ \\
\hline 4 & Mono: PM-PM-PM & $1.36 \pm 0.015^{\mathrm{hi}}$ & $8.51 \pm 0.376^{\mathrm{gh}}$ & $8.12 \pm 0.028^{h}$ & $2.62 \pm 0.069^{h}$ & $35.25 \pm 0.75^{\mathrm{ab}}$ & $80.75 \pm 1.427^{a}$ \\
\hline 5 & Mono: $\mathrm{CP}-\mathrm{CP}-\mathrm{CP}$ & $3.28 \pm 0.012^{a b c}$ & $20.51 \pm 0.303^{a b}$ & $10.30 \pm 0.306^{\mathrm{bcd}}$ & $2.70 \pm 0.096^{\mathrm{fgh}}$ & $26.4 \pm 1.21$ ef & $66.5 \pm 1.798^{\mathrm{d}}$ \\
\hline 6 & Perennial: BNH & $1.59 \pm 0.017 \mathrm{ghi}$ & $9.92 \pm 0.306^{\mathrm{fgh}}$ & $10.41 \pm 0.078^{a b c}$ & $2.90 \pm 0.08^{\operatorname{defg}}$ & $34.2 \pm 0.326^{\mathrm{ab}}$ & $76.78 \pm 1.664 \mathrm{a}^{\mathrm{bc}}$ \\
\hline 7 & Perennial: LU & $3.34 \pm 0.03^{\mathrm{ab}}$ & $20.62 \pm 1.438^{\mathrm{ab}}$ & $10.49 \pm 0.06^{\mathrm{abc}}$ & $3.20 \pm 0.122 \mathrm{bc}$ & $25.87 \pm 0.2^{\text {ef }}$ & $65.69 \pm 4.942^{\mathrm{d}}$ \\
\hline 8 & Perennial: DS & $2.96 \pm 0.007^{\mathrm{d}}$ & $18.53 \pm 0.589^{c}$ & $9.04 \pm 0.017^{\mathrm{fg}}$ & $2.92 \pm 0.073^{\mathrm{def}}$ & $29.2 \pm 1.299$ cde & $69.5 \pm 2.332^{\mathrm{cd}}$ \\
\hline 9 & Perennial: SE & $3.57 \pm 0.02^{\mathrm{a}}$ & $22.32 \pm 1.267^{\mathrm{a}}$ & $10.98 \pm 0.275^{\mathrm{ab}}$ & $3.78 \pm 0.066^{\mathrm{a}}$ & $23.05 \pm 0.914^{\mathrm{f}}$ & $62.93 \pm 0.826^{d}$ \\
\hline 10 & $\begin{array}{l}\text { Mixed: } \mathrm{MZ}+\mathrm{CP}-\mathrm{OT} \\
\quad+\mathrm{CP}-\mathrm{PM}+\mathrm{CP}\end{array}$ & $1.95 \pm 0.349^{\mathrm{f}}$ & $12.19 \pm 0.892^{\mathrm{e}}$ & $9.48 \pm 0.738^{\text {def }}$ & $2.83 \pm 0.127^{\text {efgh }}$ & $32.91 \pm 1.293^{b c}$ & $75.51 \pm 2.919^{a b c}$ \\
\hline 11 & $\begin{array}{l}\text { Mixed: SG + CP-MZ } \\
\quad+\text { CP-PM + CP }\end{array}$ & $1.86 \pm 0.167^{\mathrm{fg}}$ & $11.57 \pm 0.59$ ef & $9.41 \pm 0.60^{\text {ef }}$ & $2.99 \pm 0.029$ cde & $32.59 \pm 1.296^{b c}$ & $76.04 \pm 5.62^{a b c}$ \\
\hline 12 & Perennial: $\mathrm{BNH}+\mathrm{CP}$ & $2.91 \pm 0.012^{d}$ & $18.2 \pm 1.471^{\mathrm{cd}}$ & $11.22 \pm 0.572^{a}$ & $3.02 \pm 0.01^{\text {cde }}$ & $26.93 \pm 0.992 \mathrm{de}$ & $67.57 \pm 2.115^{\mathrm{d}}$ \\
\hline 13 & Perennial: BNH + LU & $3.01 \pm 0.111^{\mathrm{cd}}$ & $18.78 \pm 0.912 \mathrm{bc}$ & $10.86 \pm 0.301 \mathrm{abc}$ & $3.28 \pm 0.042^{b}$ & $26.94 \pm 1.309$ de & $67.09 \pm 2.048^{\mathrm{d}}$ \\
\hline 14 & Perennial: $\mathrm{BNH}+\mathrm{DS}$ & $2.61 \pm 0.029$ e & $16.32 \pm 0.664^{d}$ & $10.07 \pm 0.279$ cde & $3.05 \pm 0.07$ bcde & $30.37 \pm 1.292^{\mathrm{cd}}$ & $70.57 \pm 1.467^{\mathrm{bcd}}$ \\
\hline \multirow[t]{3}{*}{15} & Perennial: BNH + SE & $3.16 \pm 0.09 \mathrm{bcd}$ & $19.75 \pm 0.709^{b c}$ & $11.18 \pm 0.332^{\mathrm{a}}$ & $3.77 \pm 0.084^{\mathrm{a}}$ & $25.82 \pm 0.317^{\text {ef }}$ & $65.31 \pm 2.926^{\mathrm{d}}$ \\
\hline & SEM & 0.10 & 0.66 & 0.29 & 0.09 & 1.31 & 2.67 \\
\hline & $\operatorname{LSD}(p=0.05)$ & 0.30 & 1.91 & 0.85 & 0.25 & 3.78 & 7.75 \\
\hline
\end{tabular}

SEM: Standard Error of Means; LSD: Least Significant Difference. Superscript represents treatment means with similar alphabets do not differ significantly at LSD = 0.05 probability $(p)$ level. Note: MZ, Maize; SG, Sorghum; OT, Oats; PM, Pearl millet; CP, Cowpea; BNH, Bajra Napier Hybrid; LU, Lucerne; DS, Desmanthus; SE, Sesbania.

Sesbania grown as a monocrop and as an intercrop with Bajra-Napier hybrid has shown significantly higher fat concentration in its fodder (3.78\% and $3.77 \%$, respectively), closely followed by lucerne as a monocrop (3.20\%) and as an intercrop with Bajra-Napier hybrid (3.28\%), indicating higher gross energy from these cropping systems fodder. Conversely, monocrop cereals viz., pearl millet and maize throughout the year showed significantly lower ether extractable fat concentration $(2.62 \%$ and $2.67 \%$, respectively), indicating lower quality fodder from these crops (Table 3 ).

Carbohydrates constitute both fibrous (T-CHO) and non-fibrous carbohydrates (NF$\mathrm{CHO}$ ). The pure culture of cereals has shown higher NF-CHO concentration over both monocrop legumes, as well as cereal + legume intercropping systems, and ranged from $36.90 \%$ in oat fodder to $34.19 \%$ in sorghum fodder. Correspondingly, significantly higher $\mathrm{T}-\mathrm{CHO}$ concentration was noticed in pearl millet $(80.75 \%)$ and sorghum $(80.55 \%)$ grown as monocrops (Table 3). However, these crops were found statistically at par with other monocrop cereal fodder crops viz., oats, maize, the Bajra-Napier hybrid, and crop mixtures such as fodder sorghum + cowpea-fodder maize + cowpea-pearl millet + cowpea, and fodder maize + cowpea-fodder oat + cowpea-pearl millet + cowpea. Interestingly, improved forage quality in terms of NF-CHO and T-CHO concentration was also noticed in the present study when legume crops, viz., cowpea, lucerne, sesbania and desmanthus, were grown as an intercrop with Bajra-Napier hybrid over their monocropping.

\subsection{Fiber Fractions}

Fiber fractions and fodder quality are indirectly related. The fodder with less fiber concentration is considered superior quality, as the higher the fiber fractions, the lower the digestibility and energy from fodder. Cereal crops grown as monocrops, except oats, have recorded significantly higher crude fiber (CF) compared to legume crops, with the highest in sorghum (34.48\%) followed by maize (31.95\%). However, among the monocrop legumes, desmanthus fodder had higher CF concentration of $28.70 \%$ followed by cowpea $(27.62 \%)$. Similarly, in perennial intercropping systems and crop mixtures, it was ranged from $27.02 \%$ in Bajra-Napier hybrid + sesbania perennial system to a maximum of $30.38 \%$ in fodder sorghum + cowpea-fodder maize + cowpea-pearl millet + cowpea crop mixture (Table 4). 
Table 4. Crude fiber (CF), acid detergent fiber (ADF), neutral detergent fiber (NDF), dry matter intake (DMI), digestible dry matter (DDM), and relative feed value (RFV) in biomass as influenced by different cropping system treatments.

\begin{tabular}{|c|c|c|c|c|c|c|c|}
\hline $\begin{array}{l}\text { T. } \\
\text { No. }\end{array}$ & Abbreviations & CF (\%) & ADF (\%) & NDF (\%) & DDI (\%) & DDM (\%) & RFV (\%) \\
\hline 1 & Mono: MZ-MZ-MZ & $31.95 \pm 1.122^{\mathrm{ab}}$ & $40.79 \pm 0.214^{a b}$ & $63.58 \pm 0.919^{a}$ & $1.89 \pm 0.0^{\text {ef }}$ & $57.12 \pm 2.974 \mathrm{fg}$ & $83.56 \pm 7.461^{h}$ \\
\hline 2 & Mono: SG-SG-SG & $34.48 \pm 1.123^{a}$ & $39.66 \pm 2.512^{a b c}$ & $62.79 \pm 1.243^{\mathrm{ab}}$ & $1.91 \pm 0.006^{\mathrm{ef}}$ & $58.01 \pm 0.242$ defg & $85.92 \pm 3.127^{h}$ \\
\hline 3 & Mono: OT-OT-OT & $27.62 \pm 0.774^{c}$ & $31.22 \pm 1.263^{\mathrm{e}}$ & $60.14 \pm 1.978 \mathrm{abc}$ & $1.99 \pm 0.003$ ef & $64.58 \pm 0.619 \mathrm{a}$ & $99.89 \pm 5.987$ efg \\
\hline 4 & Mono: PM-PM-PM & $30.52 \pm 0.671^{b c}$ & $39.75 \pm 0.108^{a b c}$ & $60.28 \pm 1.233^{a b c}$ & $1.99 \pm 0.006^{\mathrm{ef}}$ & $57.93 \pm 0.084$ efg & $89.41 \pm 6.166^{\mathrm{gh}}$ \\
\hline 5 & Mono: $\mathrm{CP}-\mathrm{CP}-\mathrm{CP}$ & $27.62 \pm 2.424^{c}$ & $36.19 \pm 0.087^{b c d}$ & $47.72 \pm 1.414^{\mathrm{f}}$ & $2.51 \pm 0.003^{a b c}$ & $60.71 \pm 1.389^{\text {bcde }}$ & $118.34 \pm 8.404^{\mathrm{bcd}}$ \\
\hline 6 & Perennial: BNH & $30.08 \pm 0.246^{\mathrm{bc}}$ & $42.26 \pm 1.107^{\mathrm{a}}$ & $64.19 \pm 0.983^{a}$ & $1.87 \pm 0.006^{\mathrm{f}}$ & $55.98 \pm 0.217^{g}$ & $81.11 \pm 5.846^{h}$ \\
\hline 7 & Perennial: LU & $27.42 \pm 2.338^{c}$ & $34.82 \pm 1.097 \mathrm{de}$ & $43.47 \pm 1.481^{\mathrm{f}}$ & $2.76 \pm 0.003^{\mathrm{a}}$ & $61.78 \pm 0.094^{a b}$ & $132.17 \pm 5.142^{\mathrm{a}}$ \\
\hline 8 & Perennial: DS & $28.70 \pm 0.848^{b c}$ & $35.80 \pm 1.389$ cde & $44.06 \pm 1.215^{\mathrm{f}}$ & $2.72 \pm 0.006^{\mathrm{ab}}$ & $61.02 \pm 1.470^{b c}$ & $128.81 \pm 8.409^{a b}$ \\
\hline 9 & Perennial: SE & $27.29 \pm 0.088^{c}$ & $39.05 \pm 0.212^{\mathrm{abcd}}$ & $48.74 \pm 1.536^{\text {ef }}$ & $2.46 \pm 0.006^{\mathrm{bc}}$ & $58.48 \pm 0.163^{\text {cdefg }}$ & $111.58 \pm 2.492$ de \\
\hline 10 & $\begin{array}{l}\text { Mixed: } \mathrm{MZ}+\mathrm{CP}-\mathrm{OT} \\
\quad+\mathrm{CP}-\mathrm{PM}+\mathrm{CP}\end{array}$ & $28.75 \pm 2.087^{b c}$ & $36.06 \pm 3.569^{\mathrm{cd}}$ & $55.48 \pm 2.523^{\text {cde }}$ & $2.16 \pm 0.092 \mathrm{de}$ & $60.81 \pm 0.188^{\text {bcd }}$ & $101.94 \pm 6.056^{\text {ef }}$ \\
\hline 11 & $\begin{array}{l}\text { Mixed: SG + CP-MZ } \\
\quad+\mathrm{CP}-\mathrm{PM}+\mathrm{CP}\end{array}$ & $30.38 \pm 2.066^{\mathrm{bc}}$ & $38.04 \pm 1.605^{a b c d}$ & $56.05 \pm 1.422^{\mathrm{bcd}}$ & $2.14 \pm 0.081$ ef & $59.26 \pm 0.129$ bcdef & $98.34 \pm 2.809 \mathrm{fg}$ \\
\hline 12 & Perennial: $\mathrm{BNH}+\mathrm{CP}$ & $27.83 \pm 1.177^{c}$ & $36.20 \pm 1.703^{\mathrm{bcd}}$ & $48.96 \pm 5.984$ def & $2.45 \pm 0.127^{b c}$ & $60.7 \pm 0.205^{\text {bcde }}$ & $115.32 \pm 5.968^{\mathrm{cd}}$ \\
\hline 13 & Perennial: BNH + LU & $27.65 \pm 0.831^{c}$ & $34.53 \pm 2.604^{\mathrm{de}}$ & $45.85 \pm 4.653^{f}$ & $2.62 \pm 0.312^{a b c}$ & $62 \pm 0.0^{a b}$ & $125.77 \pm 2.877 \mathrm{abc}$ \\
\hline 14 & Perennial: BNH + DS & $28.39 \pm 0.150^{b c}$ & $35.78 \pm 0.116^{\text {cde }}$ & $46.35 \pm 1.636^{f}$ & $2.59 \pm 0.096^{a b c}$ & $61.03 \pm 0.091^{b c}$ & $122.49 \pm 6.739 \mathrm{abcd}$ \\
\hline \multirow[t]{3}{*}{15} & Perennial: $\mathrm{BNH}+\mathrm{SE}$ & $27.02 \pm 0.768^{c}$ & $38.57 \pm 0.042^{a b c d}$ & $49.29 \pm 2.707^{\mathrm{def}}$ & $2.43 \pm 0.064^{\mathrm{cd}}$ & $58.85 \pm 0.036^{\text {cdef }}$ & $111.05 \pm 3.002 \mathrm{de}$ \\
\hline & SEM & 1.24 & 1.60 & 2.52 & 0.10 & 0.97 & 4.25 \\
\hline & $\operatorname{LSD}(p=0.05)$ & 3.59 & 4.64 & 7.29 & 0.28 & 2.82 & 12.31 \\
\hline
\end{tabular}

SEM: Standard Error of Means; LSD: Least Significant Difference. Superscript represents treatment means with similar alphabets do not differ significantly at LSD = 0.05 probability $(p)$ level. Note: MZ, Maize; SG, Sorghum; OT, Oats; PM, Pearl millet; CP, Cowpea; BNH, Bajra Napier Hybrid; LU, Lucerne; DS, Desmanthus; SE, Sesbania.

Significantly lower acid detergent fiber (ADF) concentration was noticed in monocrop oats $(31.22 \%)$, but this value was found to be on par with perennial legumes grown as monocrops viz., lucerne (34.82\%) and desmanthus (35.80\%), and both grown as an intercrop with Bajra-Napier hybrid (34.53\% and 35.78\%, respectively). On the other hand, significantly lower neutral detergent fiber (NDF) concentration when compared to cereal fodder crops was found in monocrop legumes viz., lucerne (43.47\%), desmanthus (44.06\%), cowpea $(47.72 \%)$, sesbania $(48.74 \%)$, and these legumes as an intercrop with Bajra-Napier hybrid ( $45.85 \%, 46.35 \%, 48.96 \%$ and $49.29 \%$, respectively) (Table 4 ).

\subsection{Drymatter Intake, Digestible Drymatter, and Relative Feed Value}

The fodder quality in terms of fiber concentration determines the intake, digestibility, and feed value of the fodder. In the present study, due to their lower fiber and succulent nature, monocrop lucerne fodders have shown significantly lower DMI $(2.76 \%)$ but were statistically on par with perennial desmanthus monocrops (2.72\%) and Bajra-Napier hybrid + lucerne system (2.62\%). Due to higher DMI, pure crop of lucerne $(61.78 \%)$ and BajraNapier hybrid + lucerne system (62\%) showed higher DDM. Moreover, due to its more succulent nature, significantly higher DDM was recorded in monocrop oats fodder (64.58\%). Conversely, significantly lower DMI (1.87\%) and DDM (55.98\%) was recorded in the BajraNapier hybrid monocropping system due to higher fiber fractions. Overall, legumes as monocrops and cereal-legume intercropping systems showed significantly higher RFV compared to monocultures of cereal crops. The maximum RFV of $132.17 \%$ was recorded with monocrop lucerne, but this value was found to be on par with monocrop desmanthus $(128.81 \%)$ and perennial legumes viz., lucerne and desmanthus grown as an intercrop with the Bajra-Napier hybrid (125.77 and $122.49 \%$, respectively). On the other hand, due to lower DMI and DDM, pure cropping of the Bajra-Napier hybrid (81.11\%) and maize $(83.56 \%)$ had lower RFV (Table 4).

\subsection{Yield of Quality Traits}

The yield of quality traits was significantly influenced by different year-round fodder cropping system modules (Table 5). Perennial Bajra-Napier hybrids intercropped with legumes viz., sesbania, lucerne, and cowpea have achieved substantially higher CPY (6.18, 6.02 , and $5.60 \mathrm{t} /$ ha/year, respectively), TDCPY $(5.93,5.77$, and $5.37 \mathrm{t} / \mathrm{ha} /$ year, respectively), ash $(3.50,3.48$, and $3.46 \mathrm{t} / \mathrm{ha}$ /year, respectively), fat $(1.18,1.05$, and $0.93 \mathrm{t} / \mathrm{ha} /$ year, respec- 
tively) and T-CHO (20.3, 21.5, and $20.8 \mathrm{t} / \mathrm{ha}$ /year, respectively). However, the Bajra-Napier hybrid intercropped with lucerne (8.63 $\mathrm{t} / \mathrm{ha}$ /year) achieved significantly higher NF-CHO yield followed by pure crop of Bajra-Napier hybrid ( $8.47 \mathrm{t} /$ ha/year). Irrespective of quality traits, both cereals and legumes grown as a monocrop recorded lower yield in comparison to perennial cereal-legume intercropping systems in the present study.

Table 5. Quality yield of crude protein (CP), total dry crude protein (TDCP), ash, fat, total carbohydrate (T-CHO), and non-fiber carbohydrate (NF-CHO) in biomass as influenced by different cropping system treatments.

\begin{tabular}{|c|c|c|c|c|c|c|c|}
\hline T. No. & Abbreviations & $\begin{array}{c}\mathrm{CP} \\
\text { (t/ha/Year) }\end{array}$ & $\begin{array}{c}\text { TDCP } \\
\text { (t/ha/Year) }\end{array}$ & $\begin{array}{c}\text { Ash } \\
(\mathrm{t} / \mathrm{ha} / \text { Year })\end{array}$ & $\begin{array}{c}\text { Fat } \\
(\mathrm{t} / \mathrm{ha} / \text { Year })\end{array}$ & $\begin{array}{l}\text { NF-CHO } \\
\text { (t/ha/Year) }\end{array}$ & $\begin{array}{l}\text { T-CHO } \\
\text { (t/ha/Year) }\end{array}$ \\
\hline 1 & Mono: MZ-MZ-MZ & $2.13 \pm 0.122^{\text {efg }}$ & $1.87 \pm 0.113^{\mathrm{fg}}$ & $2.19 \pm 0.096^{c}$ & $0.57 \pm 0.009^{\mathrm{fg}}$ & $7.06 \pm 0.557^{\mathrm{bcd}}$ & $16.60 \pm 0.520$ ef \\
\hline 2 & Mono: SG-SG-SG & $1.69 \pm 0.085^{g}$ & $1.44 \pm 0.075^{g}$ & $1.72 \pm 0.029 \mathrm{de}$ & $0.65 \pm 0.064^{\text {ef }}$ & $7.12 \pm 0.338^{\mathrm{bcd}}$ & $16.80 \pm 0.214^{\mathrm{e}}$ \\
\hline 3 & Mono: OT-OT-OT & $1.73 \pm 0.139 \mathrm{fg}$ & $1.48 \pm 0.112^{g}$ & $1.41 \pm 0.025^{\mathrm{e}}$ & $0.51 \pm 0.023 \mathrm{gh}$ & $6.16 \pm 0.159 \mathrm{de}$ & $13.00 \pm 0.368^{\mathrm{gh}}$ \\
\hline 4 & Mono: PM-PM-PM & $1.52 \pm 0.151^{\mathrm{g}}$ & $1.27 \pm 0.109^{g}$ & $1.45 \pm 0.015^{\mathrm{e}}$ & $0.47 \pm 0.021^{\mathrm{h}}$ & $6.29 \pm 0.407^{\mathrm{cd}}$ & $14.40 \pm 0.407^{\mathrm{fg}}$ \\
\hline 5 & Mono: $\mathrm{CP}-\mathrm{CP}-\mathrm{CP}$ & $3.19 \pm 0.049^{\mathrm{d}}$ & $2.89 \pm 0.277^{\mathrm{d}}$ & $1.60 \pm 0.029 \mathrm{e}$ & $0.42 \pm 0.056^{\mathrm{h}}$ & $4.11 \pm 0.105^{\mathrm{f}}$ & $10.40 \pm 0.388^{\mathrm{i}}$ \\
\hline 6 & Perennial: BNH & $2.45 \pm 0.110$ def & $2.18 \pm 0.195^{\text {ef }}$ & $2.57 \pm 0.125^{b}$ & $0.72 \pm 0.037^{\text {de }}$ & $8.47 \pm 0.266^{\mathrm{a}}$ & $19.00 \pm 0.347^{\mathrm{bcd}}$ \\
\hline 7 & Perennial: LU & $3.98 \pm 0.309^{c}$ & $3.66 \pm 0.252^{c}$ & $2.00 \pm 0.015^{\mathrm{cd}}$ & $0.61 \pm 0.018^{f}$ & $4.93 \pm 0.180^{\text {ef }}$ & $12.50 \pm 0.343 \mathrm{ghi}$ \\
\hline 8 & Perennial: DS & $2.90 \pm 0.107^{\mathrm{d}}$ & $2.62 \pm 0.198$ de & $1.42 \pm 0.169^{\mathrm{e}}$ & $0.46 \pm 0.010^{\mathrm{h}}$ & $4.58 \pm 0.148^{\mathrm{f}}$ & $10.90 \pm 0.448^{h i}$ \\
\hline 9 & Perennial: SE & $4.73 \pm 0.301^{b}$ & $4.39 \pm 0.202^{b}$ & $2.33 \pm 0.318^{b c}$ & $0.80 \pm 0.027^{\mathrm{d}}$ & $4.89 \pm 0.209^{f}$ & $13.30 \pm 0.811^{g}$ \\
\hline 10 & $\begin{array}{l}\text { Mixed: } \mathrm{MZ}+\mathrm{CP}-\mathrm{OT} \\
\quad+\mathrm{CP}-\mathrm{PM}+\mathrm{CP}\end{array}$ & $2.75 \pm 0.304 \mathrm{de}$ & $2.60 \pm 0.160$ de & $2.18 \pm 0.155^{c}$ & $0.64 \pm 0.054$ ef & $7.47 \pm 0.768^{a b c}$ & $17.30 \pm 1.208^{\mathrm{de}}$ \\
\hline 11 & $\begin{array}{l}\text { Mixed: SG + CP-MZ } \\
\quad+\text { CP-PM + CP }\end{array}$ & $2.82 \pm 0.197^{\mathrm{de}}$ & $2.67 \pm 0.199$ de & $2.30 \pm 0.171^{b c}$ & $0.73 \pm 0.010^{\text {de }}$ & $7.96 \pm 0.862^{\mathrm{ab}}$ & $18.50 \pm 0.828^{\text {cde }}$ \\
\hline 12 & Perennial: $\mathrm{BNH}+\mathrm{CP}$ & $5.60 \pm 0.820^{\mathrm{a}}$ & $5.37 \pm 0.127^{\mathrm{a}}$ & $3.46 \pm 0.208^{\mathrm{a}}$ & $0.93 \pm 0.015^{\mathrm{c}}$ & $8.29 \pm 0.314^{\mathrm{ab}}$ & $20.80 \pm 0.693^{\mathrm{ab}}$ \\
\hline 13 & Perennial: $\mathrm{BNH}+\mathrm{LU}$ & $6.02 \pm 0.216^{\mathrm{a}}$ & $5.77 \pm 0.500^{a}$ & $3.48 \pm 0.049^{a}$ & $1.05 \pm 0.025^{b}$ & $8.63 \pm 0.765^{\mathrm{a}}$ & $21.50 \pm 0.532^{\mathrm{a}}$ \\
\hline 14 & Perennial: $\mathrm{BNH}+\mathrm{DS}$ & $4.17 \pm 0.222 \mathrm{bc}$ & $3.98 \pm 0.309 b c$ & $2.57 \pm 0.196^{b}$ & $0.78 \pm 0.024^{\mathrm{d}}$ & $7.76 \pm 0.189 \mathrm{ab}$ & $18.10 \pm 1.335 \mathrm{de}$ \\
\hline \multirow[t]{3}{*}{15} & Perennial: BNH + SE & $6.18 \pm 0.111^{\mathrm{a}}$ & $5.93 \pm 0.210^{a}$ & $3.50 \pm 0.116^{\mathrm{a}}$ & $1.18 \pm 0.044^{\mathrm{a}}$ & $8.08 \pm 0.327^{a b}$ & $20.30 \pm 1.565^{a b c}$ \\
\hline & SEM & 0.26 & 0.21 & 0.13 & 0.03 & 0.43 & 0.76 \\
\hline & $\operatorname{LSD}(p=0.05)$ & 0.74 & 0.61 & 0.38 & 0.10 & 1.24 & 2.20 \\
\hline
\end{tabular}

SEM: Standard Error of Means; LSD: Least Significant Difference. Superscript represents treatment means with similar alphabets do not differ significantly at LSD $=0.05$ probability $(p)$ level. Note: MZ, Maize; SG, Sorghum; OT, Oats; PM, Pearl millet; CP, Cowpea; BNH, Bajra Napier Hybrid; LU, Lucerne; DS, Desmanthus; SE, Sesbania.

\subsection{Sustainable Yield Index and Land Use Efficiency}

Based on the productivity, the sustainable yield index (SYI) values were determined for the different cropping systems, and found higher SYI values for perennial intercropping systems in our present study (Figure 3). The perennial Bajra-Napier hybrid + lucerne system had a higher SYI value of 0.97 , followed by Bajra-Napier hybrid + cowpea ( 0.87$)$ and Bajra-Napier hybrid + sesbania perennial systems (0.74). However, monocropping of cowpea, desmanthus, and oats showed negative SYI values $(-0.06,-0.04$, and -0.01 , respectively). Higher LUE was also achieved when the Bajra-Napier hybrid was intercropped with legumes (cowpea, lucerne, desmanthus, and sesbania) and in monocropping desmanthus, sesbania, the Bajra-Napier hybrid, and lucerne, each with $98.63 \%$ (Figure 3). In contrast, annual intercropping systems and seasonal fodder crops (maize, sorghum, oats, bajra, and cowpea) showed lower LUE, with the least in the cowpea $(41.10 \%)$ and oats $(45.21 \%)$ systems. 


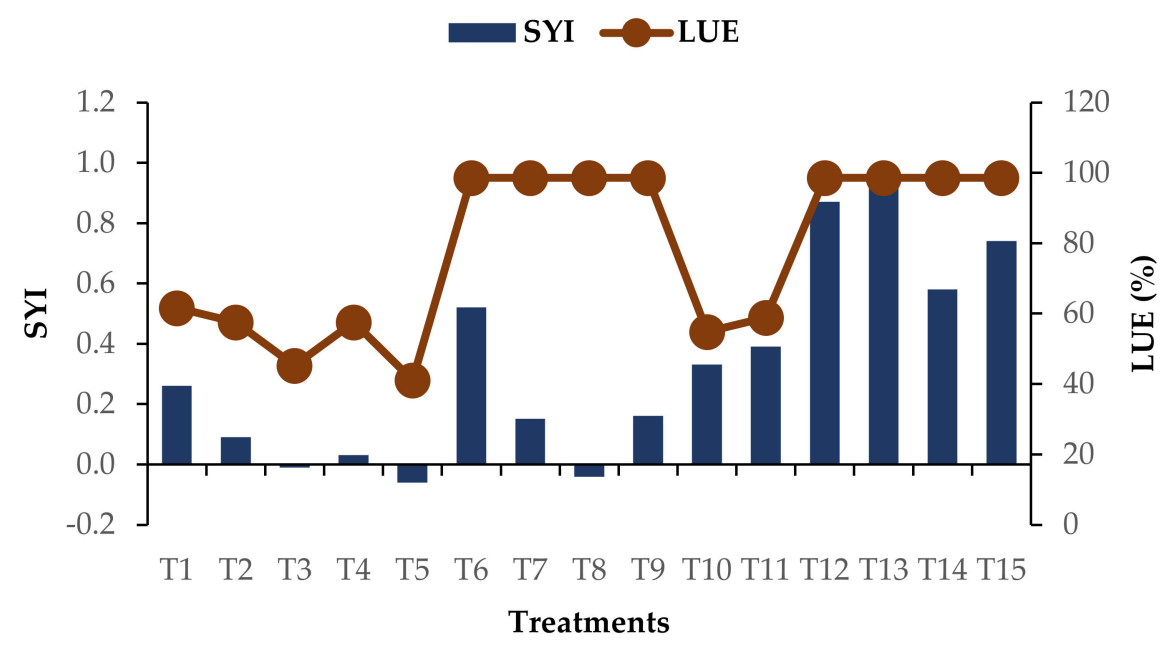

Figure 3. Sustainability yield index (SYI) and light use efficiency (LUE) as influenced by different cropping system treatments. For treatment details please see Table 1.

\section{Discussion}

\subsection{Biomass Yield}

Accessibility of quality green fodder is challenging for the livestock sector due to poor quality, i.e., deficiency in required amount of protein, minerals, and energy, leading to lower productivity of livestock [20]. To meet the current green fodder needs, intensive fodder cropping systems which promote the productivity per unit area and specified time are necessary [21]. In the present study, intercropping of perennial Bajra-Napier hybrid with legume fodder crops viz., cowpea, lucerne, and sesbania had shown significant improvement in the biomass yield over monocropping systems and other crop mixtures. The complementary nature of intercropped cereals and legumes might have resulted in efficient utilization of available resources such as nutrients and water, which eventually improved the fodder yield through better growth parameters [22]. In addition, legumes also supply $\mathrm{N}$ through biological $\mathrm{N}$ fixation that will favor the enhancement of plant growth and development [23]. The higher GFY of the component crops associated with perennial cropping systems resulted in higher DMY, even with considerable variation in dry matter concentration. Perennial intercropping systems such as Bajra-Napier hybrid + cowpea; guinea grass + cowpea [2]; and Bajra-Napier hybrid (BNH-10) + desmanthus at 1:5 row proportions produced higher GFY [21]. Increased DMY with Napier grass and herbaceous perennial legume intercropping systems were reported by Menbere et al. [24] from research in Ethiopia. These biomass results agree with findings of Eskandari [25], Deore et al. [26], and Shekara et al. [27]. The importance and superiority of Napier grass for livestock feed has been demonstrated in several countries around the world, particularly in Kenya [28] and other parts of East and Central Africa [29]. The more fibrous nature of the stems and considerably lower leaf to stem ratio in the perennial intercropping systems resulted in higher DM concentration in our study. Rajput et al. [30] and Iyanar et al. [31] reported higher DM content of fodder in sorghum grown as a monocrop. In this study, we did not statistically compare individual crop components' biomass yield in intercropping with the monocropping system on similar land area basis. Our visual observation of the crop growth did not show any differences. The response will depend on the available aboveand below-ground resources. However, further research would be needed to compare the productivity of each crop under different systems (monocropping, intercropping, or mixed cropping).

\subsection{Quality Traits and its Yield}

In general, cereals are the main source of energy (carbohydrates) while legumes are rich in CP [21]. Thus, adopting both cereal and legumes as component crops in 
cropping systems provides nutritionally rich fodder for the livestock. In the present study, monocrop legumes fodders have shown higher $\mathrm{N}$ and $\mathrm{CP}$ concentration. However, when they cultivated with cereal crops in intercropping systems, the improvement in the $\mathrm{N}$ and $\mathrm{CP}$ content was noticed as compared to monocrop with cereal fodder. Symbiotic biological $\mathrm{N}$ fixation by legumes in the cereal legume intercropping systems improves nutrient uptake [32], which may have resulted in more availability of $\mathrm{N}$, and thereby more $\mathrm{N} \%$ in biomass. Subsequently, higher $\mathrm{N}$ has resulted in better protein biosynthesis [33]. Studies conducted by Yadav et al. [34] revealed the higher CP in legumes fodder when they were grown as monocrops. Singh et al. [1] and Tamta et al. [10] found the improvement in the CP concentration in Bajra-Napier hybrid + cowpea and maize + cowpea intercropping systems than monocrop Bajra-Napier hybrid and maize fodder, respectively.

Similar to $\mathrm{CP}$, the ash concentration of fodder was found to be improved under perennial Bajra-Napier hybrid + legumes (cowpea and sesbania) cropping systems. Higher growth and dry matter accumulation by the intercropping systems due to the higher availability of $\mathrm{N}$ and other nutrients resulted in a higher ash concentration. This was further evident by the strong positive correlation of the ash content with $\mathrm{CP}\left(\mathrm{r}^{2}=0.71\right)$ and the $\mathrm{N}$ concentration $\left(\mathrm{r}^{2}=0.71\right)$ of the fodder (Figure 4$)$. Higher ash content under guinea grass + cowpea intercropping system was earlier reported by Singh et al. [1]. Likewise, Rasool et al. [35] also reported the higher ash content under cereal-legume intercropping systems. These above-presented results are also concordant with the earlier findings of Akhtar et al. [36], Ayub et al. [37], Iqbal et al. [8], and Uher et al. [38].

Fat concentration is one of the important quality traits that determine the gross energy of various forages and feeds, as fat yields over $9 \mathrm{Kcal} / \mathrm{g}$ while proteins and carbohydrates yield about $5 \mathrm{Kcal} / \mathrm{g}$ [39]. In our present study, monoculture of perennial legumes and as an intercrop with the Bajra-Napier hybrid had significantly higher fat concentration than other cropping systems. Nadeem et al. [40] reported improved fat content by $3.3 \%$ in maize + cowpea fodder than in maize fodder alone.

In contrast to $\mathrm{N}, \mathrm{CP}$, ash, and fat concentrations, higher $\mathrm{NF}-\mathrm{CHO}$ and $\mathrm{T}-\mathrm{CHO}$ concentration was noticed in cereal fodder crops grown as pure crops. This kind of composition makes fodder inadequate for animal nutrition. Strong negative correlation of non-fiber $\mathrm{CHO}$ and total $\mathrm{CHO}$ concentration with $\mathrm{CP}\left(\mathrm{r}^{2}=-0.96\right.$ and $\left.\mathrm{r}^{2}=-0.99\right)$ and ash $\left(\mathrm{r}^{2}=-0.79\right.$ and $r^{2}=-0.80$ ) was also observed in the present study (Figure 4 ). In contrast, cereal + legume intercropping systems are viable for increasing forage quality and yield [41,42]. Interestingly, we also found improved forage quality with higher $\mathrm{CP}$, ash, fat, NF-CHO, and $\mathrm{T}-\mathrm{CHO}$ concentration when perennial Bajra-Napier hybrid was intercropped with legumes viz., cowpea, lucerne, sesbania, and desmanthus. The improved forage quality, with higher $\mathrm{CP}$ and $\mathrm{CHO}$ content under corn + soybean system and lower quality under sole cropping systems, was also reported by Baghdadi et al. [43] and Mallikarjun et al. [30]. With respect to quality traits yield, the Bajra-Napier hybrid intercropped with sesbania, lucerne, and cowpea achieved substantially higher yield in the present study. Higher DMY reflected the higher quality yield in Bajra-Napier hybrid + legume cropping systems, irrespective of quality concentrations. A higher CPY was observed in Bajra-Napier hybrid and guinea grass intercropped with cowpea [2]. Tamta et al. [10] obtained nutritionally rich quality fodder in terms of higher CP and TDCP yields with cereal-legume intercropping systems. Higher ash and ether extractable fat yields in Bajra-Napier hybrid and guinea grass intercropped with cowpea was reported by Singh et al. [1]. 


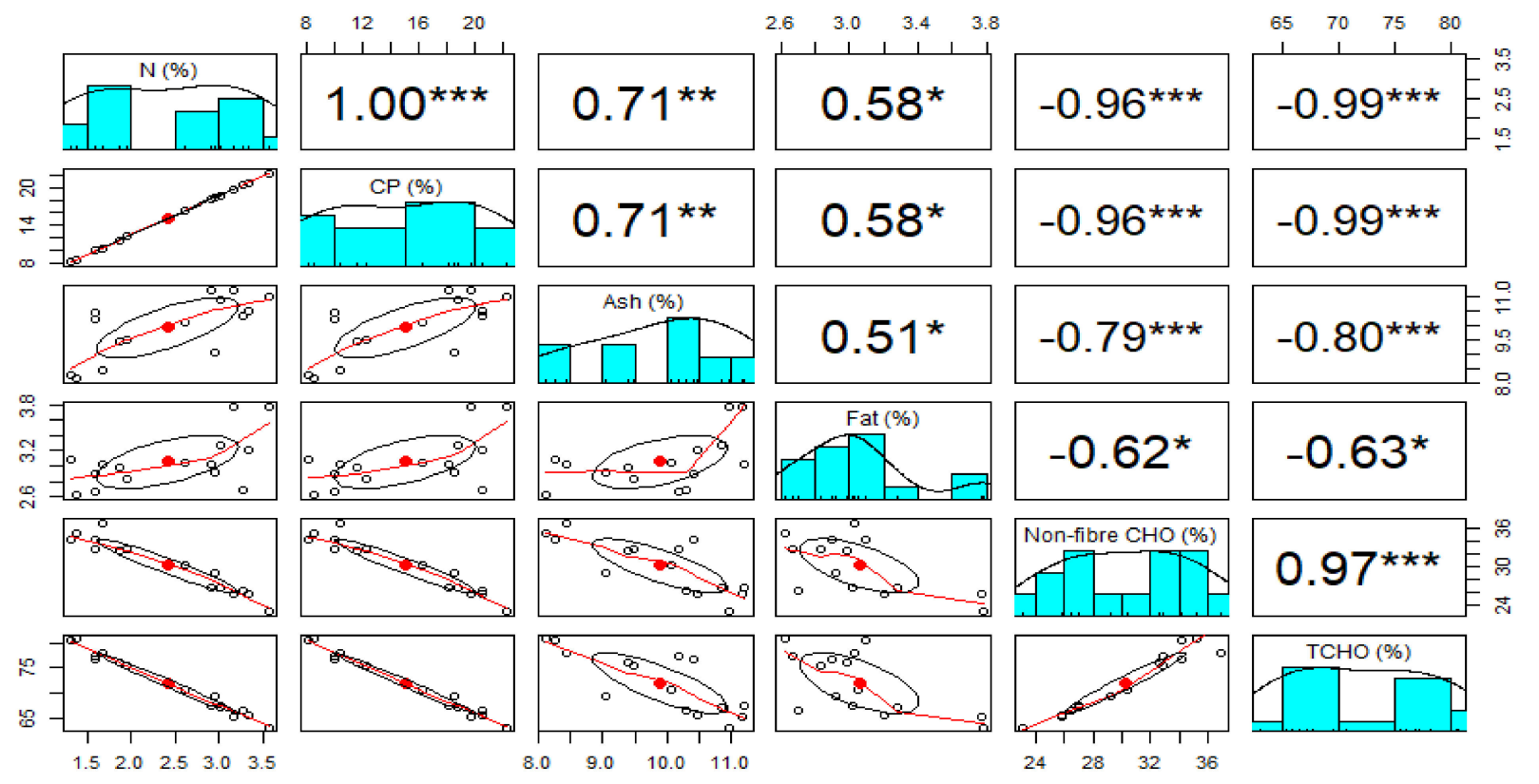

Figure 4. Relationship between quality parameters of fodder under different cropping system modules. Nitrogen (N); crude protein $(\mathrm{CP})$, ash, fat, total carbohydrate (T-CHO), and non-fiber $\mathrm{CHO}{ }^{*},{ }^{* *}$, and ${ }^{* * *}$, significant at $p<0.05,0.01$, and 0.001 , respectively.

\subsection{Fibre Fractions}

Fodder having the lower $\mathrm{CF}, \mathrm{ADF}$, and NDF with the higher $\mathrm{CP}$, ash, fat, and $\mathrm{CHO}$ is often considered as a nutritionally rich fodder for the livestock [43]. In our study, when they are grown as monocrops, cereal recorded higher $\mathrm{CF}$, while legumes recorded lower $\mathrm{CF}$. The higher dry matter concentration and better storage of photosynthates as cell wall contents during their partitioning have attributed to the higher CF [44]. Higher CF in sole cropped sorghum was reported by Akhtar et al. [36]. Previous studies also reported lower crude fiber in pearl millet + cowpea [45] and sesbania + pearl millet intercropping systems [35]. Similar to $\mathrm{CF}$, reduced ADF and NDF concentration was noticed with monocrop legumes and Bajra-Napier hybrid + legumes intercropping systems in our study. Generally, ADF and NDF are greater in grasses than legumes, thus the magnitude of this difference and the proportion of legume crops in cropping systems seemed to have impacted fiber fractions of different cropping systems. Studies on monoculture maize [5] and hybrid Napier [46] reported higher ADF and NDF. Maize intercropping with legumes boosted CP content with lowered NDF and ADF concentrations, and so improved fodder nutritive value [25,43]. Furthermore, a strong negative correlation of NDF with CP $\left(\mathrm{r}^{2}=0.91\right)$ (Figure 5) revealed that fodder with higher CP increases the palatability by lowering NDF.

\subsection{Intake, Digestibility, and Relative Feed Value of Drymatter}

The fodder with higher CP and lower fiber fractions makes it more palatable, and thereby improves the digestibility for livestock [16]. In the present study, the monoculture of legumes (cowpea, lucerne, sesbania, and desmanthus) and Bajra-Napier hybrid + legume intercropping systems have shown improved DMI and DDM compared to monocrop cereals. Legumes, both as sole crops and as an intercrop with cereals, were shown to have lower fiber content, indicating higher palatability and digestibility, leading to higher intake on a dry matter basis $[47,48]$. The lower ADF concentration and succulent nature makes fodder more palatable and easier for digestion by microbes in the rumen of livestock. The negative correlation $\left(\mathrm{r}^{2}=-1\right)$ depicts improvement of DDM with lower ADF (Figure 5). Similar results were revealed by others [48-51]. RVF reflects both intake potential and digestibility of biomass [52]. Higher CP, DMI and DDM of pure legume crops and BajraNapier hybrid + legume intercropping systems reflected in higher RFV in current study. The 
higher RFV of lucerne forage was reported by others [52,53]. Correlation values $\left(\mathrm{r}^{2}=0.86\right.$, -0.98 and 0.98 ) showed dependency of RFV on CP, NDF, and DMI of fodder, respectively (Figure 5), and these results were supported by Anil et al. [54] and Prajapati et al. [48,55].
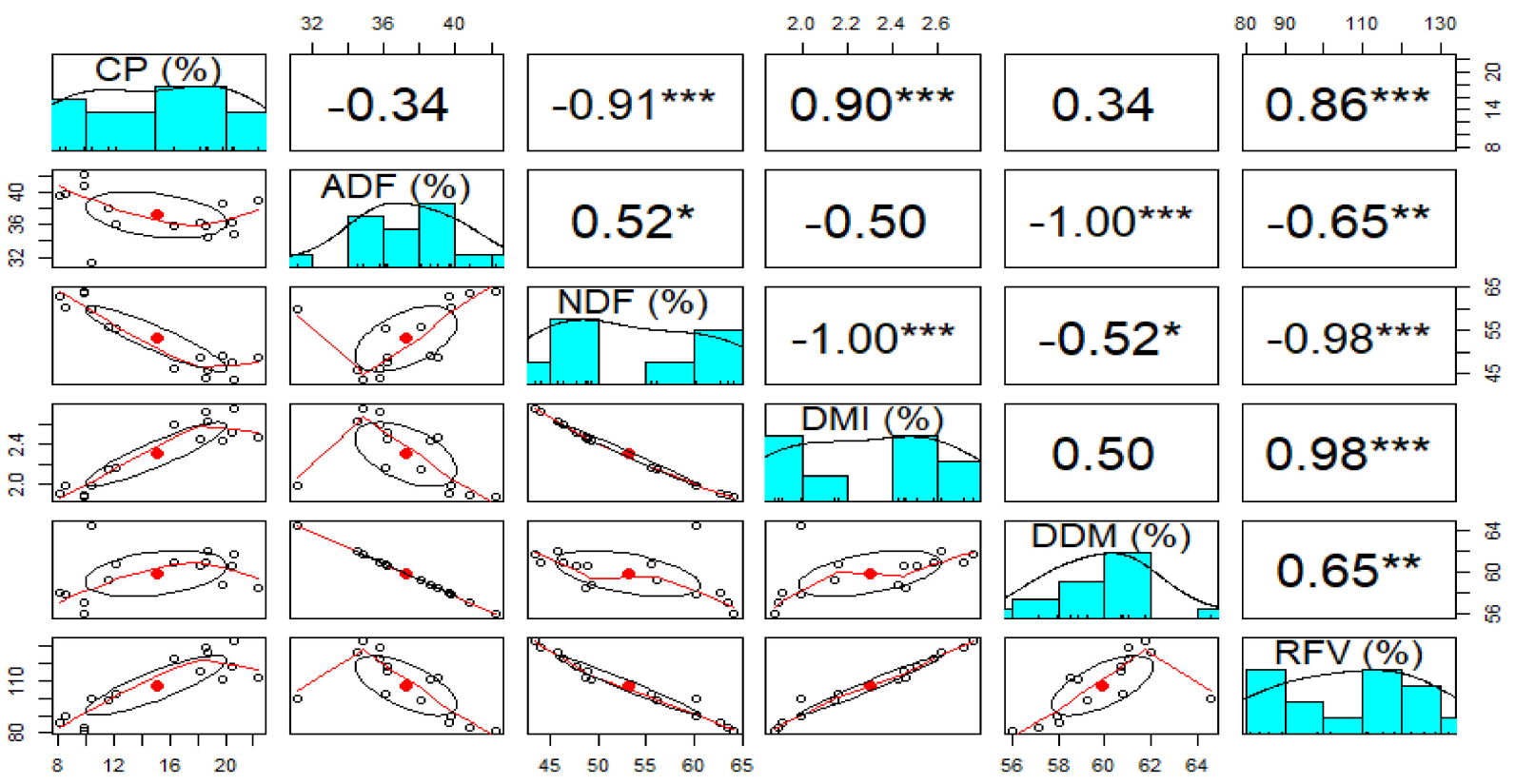

Figure 5. Relationship between quality parameters of fodder under different cropping system modules. Crude protein $(\mathrm{CP})$, acid detergent fiber (ADF), neutral detergent fiber (NDF), dry matter intake (DMI), digestible dry matter (DDM), and relative feed value (RFV). ${ }^{*}{ }^{* *}$, and ${ }^{* * *}$, significant at $p<0.05,0.01$, and 0.001 , respectively.

\subsection{Stability and Efficiency of the Systems}

In the long run, cropping system stability, in terms of the qualitative and quantitative biomass production with an effective utilization of land resources, is critical. Bajra-Napier hybrid + lucerne system was found more stable, with higher SYI (0.97), followed by BajraNapier hybrid + cowpea and Bajra-Napier hybrid + sesbania perennial systems. The higher productivity associated with these perennial intercropping systems during both the years of study resulted in higher sustainability over crop mixtures and monocrops. Higher SYI of 0.80 with hybrid Napier-hybrid Napier + berseem-hybrid Napier cropping systems, and higher SYI of perennial cereal-legume cropping system modules are in accordance with other studies [55-57]. Higher LUE (98.63\%) was also achieved with Bajra-Napier hybrid + legume intercropping systems and perennial monocrops. The greater number of days occupied by the perennial cropping system modules in a calendar year was associated with higher LUE [55,58].

The yields observed in Bajra-Napier hybrid intercropped with legumes (range 131 to $155 \mathrm{t}$ /ha/year GFY; or 25.5 to $32.1 \mathrm{t} /$ ha/year DMY) and the SYI (0.97) in our research were much higher than those observed in other fodder production systems in India. For example, maximum GFY of $118 \mathrm{t} / \mathrm{ha}$ and SYI of 0.79 was observed in maize + rice beanoat-sorghum + cowpea [58]. While the oat + fodder brassica-maize + cowpea system produced the highest GFY of $60.77 \mathrm{t} / \mathrm{ha}$ /year [56] was much lower than that which we observed in our system, but the SYI was slightly higher (0.99) compared to our study (0.97). Thus, the yields from our intercropping systems were better than the other fodder production systems.

\subsection{Strategies to Promote Fodder Production in India and Around the World}

The results from this research are applicable not only in the dry regions of the Karnataka, but also other parts of India and around the world which have similar agro-climatic conditions and a large demand for fodder. Most of the dry regions within India and 
around the world have limited opportunity to bring additional land area under fodder production without causing environmental damage. Therefore, increased production must come from increasing the productivity per unit area per unit time, using improved fodder production systems. Use of year-long fodder production with a combination of annual and perennial fodder cropping systems identified in this research will be a promising approach. The identified crops and genotypes are versatile and can be grown in different farming systems such as food-forage based cropping systems, inclusion of perennial fodder crops in orchards, plantation crops, agro-forestry, and silvi-pastural systems. The short duration of forage crops can be incorporated in rice fallows to increase cropping intensity. In addition, annual and perennial fodder crops can be grown on less productive land (e.g., waste lands, farm boundaries, problematic soils, and marshy areas). Although this research was targeted to the local context of Karnataka in India, the findings will be useful in regions around the world which have similar agroclimatic conditions. The findings of perennial crop management from this study can be tested and replicated to other agroclimatic conditions. Crop and livestock producers incorporate cereal-legume forage cropping systems to meet the demand for animal feed. The findings of this research will help them improve productivity and contribute to sustainable intensification of forage or fodder-based cropping systems. Perennial and annual forage/fodder cultivation systems in the UK [41], USA [47,59], Africa [60], and other developing countries [61] have been found beneficial for the sustainability of agroecosystem.

\section{Conclusions}

The benefit of intercropping perennial and annual forage cultivation along with a year-round cereal-legume based cropping system adds value to the livestock production system. Overall, this research has shown that the perennial cereal-legume cropping systems were more productive in terms of both green fodder and dry fodder yield than monocultures of either cereal or legume crops throughout the year. The complementary nature of component crops in intercropping systems may lead to better utilization of available resources, and thereby helps to attain improved quantity and quality of livestock feed yield. Among the various intercropping systems, the perennial cereal crop Bajra-Napier hybrid intercropped with legume crops (e.g., lucerne, cowpea, and sesbania) showed the highest forage production and quality yield in terms of crude protein, ash, and ether extractable fat. The inclusion of legumes as component crops with cereals in crop mixtures also further improved the quality due to lower fiber fractions, which resulted in increased dry matter intake, dry matter digestibility and relative feed value as they were found to be more succulent and palatable. Additionally, the perennial Bajra-Napier hybrid intercropped with lucerne, cowpea, and sesbania showed higher sustainable yield index values of 0.97 , 0.87 , and 0.74 , respectively, than other cropping system modules, demonstrating the sustainability of the modules for achieving higher yield. The result from this research provides alternative options for the livestock farmers in India and other parts of the world which have similar agro-climatic conditions to achieve both qualitative and quantitative forage for livestock production, using cultivated cereal and legume fodder crops in mixtures. The practice of promoting and adopting improved cereal-legume intercropping systems based on available resources in a region can be a pathway to sustain the feeding needs of the increasing livestock population. It can also be a way to combat the higher cost of concentrate feeds and eventually boost the livestock production by the smallholder farmers. Although the varieties of various crops used in this research were tested in a specific region of India, they can be suitable in other dry regions within and outside of India which have similar conditions. However, these varieties must be tested, and suitable agronomic packages need to be developed so they can be adopted by the producers.

Author Contributions: K.N.M.: Investigation, Formal analysis, and Manuscript writing-original draft; B.G.S.: Conceptualization, Methodology, and Supervision; S.S., P.V.V.P. and P.K.J.: Visualization and Writing-review and editing. All authors have read and agreed to the published version of the manuscript. 
Funding: This research received no external funding.

Institutional Review Board Statement: Not applicable.

Informed Consent Statement: Not applicable.

Data Availability Statement: Data is available upon request from corresponding author.

Acknowledgments: The authors are grateful to the Department of Science and Technology, New Delhi for their support for this research program. Contribution number 22-060-J from Kansas Agricultural Experiment Station.

Conflicts of Interest: The authors declare no conflict of interest.

\section{References}

1. Singh, H.N.; Kumar, M.R.; Magan, S.; Rakesh, K.; Hardev, R.; Kumar, M.V.; Manish, K. Evaluation of kharif forage crops for biomass production and nutritional parameters in Indo-Gangetic plains of India. Indian J. Anim. Nutr. 2019, 36, 25-29. [CrossRef]

2. Hindoriya, P.S.; Meena, R.K.; Rakesh, K.; Singh, M.; Ram, H.; Meena, V.K.; Ginwal, D.; Dutta, S. Productivity, and profitability of cereal-legume forages vis-a-vis their effect on soil nutrient status in Indo-Gangetic Plains. Legume Res. 2019, 42, 812-817. [CrossRef]

3. Makkar, H.P.S. Animal nutrition in a 360-degree view and a framework for future R\&D work: Towards sustainable livestock production. Anim. Prod. Sci. 2016, 56, 1561-1568. [CrossRef]

4. Chaudhary, D.P.; Ashwani, K.; Sapna, S.M.; Srivastava, P.; Kumar, R.S. Maize as Fodder? An Alternative Approach; Technical Bulletin; Directorate of Maize Research, Pusa Campus: New Delhi, India, 2012; p. 32.

5. Kumar, R.; Singh, M.; Meena, B.S.; Ram, H.; Parihar, C.M.; Kumar, S.; Yadav, M.R.; Meena, R.K.; Kumar, U.; Meena, V.K. Zinc management effects on quality and nutrient yield of fodder maize (Zea mays). Indian J. Agric. Sci. 2017, 87, $29-33$.

6. $\quad$ Roy, A.K.; Agrawal, R.K.; Bhardwaj, N.R.; Mishra, A.K.; Mahanta, S.K. Revisiting National Forage Demand and Availability Scenario; ICAR-AICRP on Forage Crops and Utilization: Jhansi, India, 2019; pp. 1-21.

7. Shekara, B.G.; Mahadevu, P.; Chikkarugi, N.M.; Manasa, N. Fodder and livestock scenario in karnataka. In Indian Fodder Scenario: Redefining State Wise Status; Indian Council of Agricultural Research-All India Coordinated Research Project on Forage Crops and Utilization: Jhansi, India, 2019; pp. 79-90. [CrossRef]

8. Iqbal, A.; Iqbal, M.N.; Akbar, N.; Waseem, M.; Khan, H.Z.; Abbas, R.N. Performance of pearl millet (Pennisetum americanum L.) forage grown in association with forage legumes under different sowing techniques. Custose Agrone Aocio Online 2013, 9, 257-269.

9. Marer, S.B.; Lingaraju, B.S.; Shashidhara, G.B. Productivity and economics of maize and pigeon pea intercropping under rainfed Northern transitional Zone of Karnataka. Karnataka J. Agric. Sci. 2007, 20, 1-3.

10. Tamta, A.; Kumar, R.; Ram, H.; Meena, R.K.; Meena, V.K.; Yadav, M.R.; Subrahmanya, D.J. Productivity and profitability of legume-cereal forages under different planting ratio and nitrogen fertilization. Legume Res. 2019, 42, 102-107. [CrossRef]

11. Reza, Z.O.; Allahdadi, I.; Mazaheri, D.; Akbari, G.A.; Jahanzad, E.; Mirshekari, M. Effect of different planting proportions and nitrogen fertilizer in intercropping forage sorghum and lima bean. Afr. J. Agric. Res. 2013, 8, 6488-6498. [CrossRef]

12. Ali, M.A.; Khan, F.H.; Ali, R.S.; Afzal, Z.; Saleem, M.T.; Azeem, M. Effect of intercropping of pearl millet and cluster bean on forage quality and quantity. J. Entomol. Zool. Stud. 2016, 4, 397-400.

13. Sudarshan Reddy, A.; Palled, Y.B. Effect of intercropped fodder cowpea on maize and system productivity in maize + fodder cowpea intercropping systems. J. Farm. Sci. 2016, 29, 265-267.

14. AOAC. The Official Methods of Analysis; Association of Official Analytical Chemists: Washington, DC, USA, 2000.

15. Iqbal, M.; Iqbal, Z.; Farooq, M.; Ali, L.; Fiaz, M. Impact of nitrogenous fertilizer on yield and quality of oat. Pak. J. Sci. 2013, $65,1-4$.

16. Horrocks, R.D.; Vallentine, J.F. Harvested Forages; Academic Press: London, UK, 1999; pp. 17-47.

17. Kumar, M.; Singh, S.R.; Jha, S.K.; Shamna, A.; Mazumdar, S.P.; Singh, A.; Kundu, D.K.; Mahapatra, B.S. System productivity, profitability and resource use efficiency of jute (Corchorus olitorius) based cropping systems in the eastern Indo-gangetic plain. Indian J. Agric. Sci. 2014, 84, 209-213.

18. Singh, R.P.; Parr, J.F.; Stewart, B.A. Dryland agriculture-Strategies for sustainability. Adv. Soil Sci. 1990, 13, 340.

19. Gomez, K.A.; Gomez, A.A. Statistical Procedure for Agriculture Research, 2nd ed.; John Willey and Sons: New York, NY, USA, 1984; p. 68.

20. Prajapati, B.; Prajapati, J.; Kumar, K.; Shrivastava, A. Determination of the relationships between quality parameters and yields of fodder obtained from intercropping systems by correlation analysis. Forage Res. 2019, 45, 219-224.

21. Patil, L.M.; Kauthale, V.K.; Bhalani, T.G.; Modi, D.J. Productivity, and economics of different forage production systems in south Gujarat conditions of India. Forage Res. 2018, 44, 14-18.

22. Dwivedi, A.; Dev, I.; Kumar, V.; Yadav, R.S.; Yadav, M.; Gupta, D.; Singh, A.; Tomar, S.S. Potential role of maize-legume intercropping systems to improve soil fertility status under smallholder farming systems for sustainable agriculture in India. Int. J. Life Sci. Biotech. Pharm. Res. 2015, 4, 145-157.

23. Deori, C.; Thakuria, K.; Kurmi, K. Effect of intercropping and planting methods on perennial grasses. Forage Res. 2019, 45, 162-164. 
24. Menbere, S.; Dejene, M.; Abreha, S. Dry matter yield and agronomic performance of herbaceous legumes intercropped with Napier grass (Pennisetum Purpureum) in the semi-arid areas of eastern Amhara region. Int. J. Recent Res. Life Sci. 2015, 2, 7-14.

25. Eskandari, H. Intercropping of maize (Zea mays) with cowpea (Vigna sinensis) and mungbean (Vigna radiata): Effect of complementarity of intercrop components on resource consumption, dry matter production and legumes forage quality. J. Basic Appl. Sci. Res. 2012, 2, 355-360.

26. Deore, S.M.; Patel, M.R.; Patel, P.M.; Patel, H.K.; Patel, U.J. Production potential of forage maize (Zea mays L.)—Cowpea (Vigna unguiculata L.) intercropping system as influenced by row ratios. Adv. Res. J. Crop. Improv. 2013, 4, 110-112.

27. Shekara, B.G.; Lohithaswa, H.C.; Chikkarugi, N.M.; Manasa, N. Fodder production potential of maize grown for baby corn and green cob in different cropping systems. Forage Res. 2015, 41, 92-94.

28. Orodho, A.B. The Role and Importance of Napier Grass in the Smallholder Dairy Industry in Kenya; Food and Agriculture Organization: Rome, Italy, 2006; p. 2011.

29. Kabirizi, J.; Muyekho, F.; Mulaa, M.; Msangi, R.; Pallangyo, B.; Kawube, G.; Zziwa, E.; Mugerwa, S.; Ajanga, S.; Lukwago, G.; et al. Napier Grass Feed Resource: Production, Constraints and Implications for Smallholder Farmers in Eastern and Central Africa; The Eastern African Agricultural Productivity Project: Naivasha, Kenya, 2015.

30. Rajput, R.D.; Paithane, V.A.; Taware, A.S.; Patil, R.P. A comparative study on the proximate and mineral composition of non-leguminous fodder crops. Biosci. Discov. 2017, 8, 890-891.

31. Iyanar, K.; Babu, C.; Kumaravadivel, N.; Kalamani, A.; Velayudham, K.; Sathia Bama, K. A high yielding multicut fodder sorghum CO-31. Electron. J. Plant. Breed. 2015, 6, 54-57.

32. Layek, J.; Das, A.; Mitran, T.; Nath, C.; Meena, R.S.; Yadav, G.S.; Shivakumar, B.G.; Kumar, S.; Lal, R. Cereal + legume intercropping: An option for improving productivity and sustaining soil health. In Legumes for Soil Health and Sustainable Management; Meena, R.S., Das, A., Yadav, G.S., Lal, R., Eds.; Springer: Singapore, 2018; pp. 347-386.

33. Mallikarjun, R.H.; Kumar, R.; Meena, R.K.; Ginwal, D. Yield and chemical composition of cowpea (Vigna unguiculata) fodder as affected by tillage practices and nitrogen management. Indian J. Anim. Nutr. 2018, 35, 333-338. [CrossRef]

34. Yadav, K.; Verma, A.; Yadav, M.K.; Choudhary, M.; Choudhary, K.M. Effect of fertilizer levels on fodder productivity and quality of multi-cut sorghum genotypes. Int. J. Bio-Resour. Stress Manag. 2019, 10, 119-123. [CrossRef]

35. Rasool, T.; Zohaib, A.; Riaz, A.; Abbas, T.; Tabassum, T.; Nadeem, M.A. Forage yield and quality in pearl millet-sesbania intercropping system under various geometrical patterns. Pak. J. Agric. Res. 2017, 30, 75-84.

36. Akhtar, M.F.; Ahmad, A.; Zamir, M.S.; Khalid, F.; Mohsin, A.U.; Afzal, M. Agro-qualitative studies on forage sorghum (Sorghum bicolor L.) sown alone and in mixture with forage legumes. Pak. J. Sci. 2013, 65, 179-185.

37. Ayub, M.; Abbas, H.; Tariq, M.; Tahir, M.; Nadeem, M.A.; Siddiqui, M.H. Periodic assessment of dry matter production and nutritional value of millet legumes mix fodder. Agric. Res. 2013, 2, 265-269. [CrossRef]

38. Uher, D.; Horvatic, I.; Jares, D.; Macesic, D. Influence of intercropping maize with cowpea on forage yield and quality. Direct Res. J. Agric. Food Sci. 2019, 7, 77-80.

39. Idris, O.A.; Wintola, O.A.; Afolayan, A.J. Comparison of the proximate composition, vitamins (ascorbic acid, $\alpha$-tocopherol and retinol), anti-nutrients (phytate and oxalate) and the GC-MS analysis of the essential oil of the root and leaf of Rumex crispus L. Plants 2019, 8, 51. [CrossRef] [PubMed]

40. Nadeem, M.A.; Iqbal, Z.; Ayub, M.; Mubeen, K.; Ibrahim, M. Effect of nitrogen application on forage yield and quality of maize sown alone and in mixture with legumes. Pak. J. Life Soc. Sci. 2009, 7, 161-167.

41. Anil, L.; Park, J.; Phipps, R.H.; Miller, F.A. Temperate intercropping of cereals for forage: A review of the potential for growth and utilization with particular reference to the UK. Grass Forage Sci. 1998, 53, 301-317. [CrossRef]

42. Sadeghpoura, A.; Jahanzada, E.; Esmaeilib, A.; Hosseinib, M.B.; Hashemia, M. Forage yield, quality and economic benefit of intercropped barley and annual medic in semi-arid conditions: Additive series. Field Crops Res. 2013, 148, 43-48. [CrossRef]

43. Baghdadi, A.; Halim, R.A.; Ghasemzadeh, A.; Ebrahimi, M.; Othman, R.; Yusof, M.M. Effect of intercropping of corn and soybean on dry matter yield and nutritive value of forage corn. Legume Res. 2016, 39, 976-981. [CrossRef]

44. Prasanthi, K. Studies on Fodder Maize and Legume Intercropping System. Ph.D. Thesis, Acharya N.G. Ranga Agricultural University, Hyderabad, India, 2012.

45. Islam, N.; Zamir, M.S.; Din, S.M.; Farooq, U.; Arshad, H.; Bilal, A.; Sajjad, M.T. Evaluating the intercropping of millet with cowpea for forage yield and quality. Am. J. Plant. Sci. 2018, 9, 1781-1793. [CrossRef]

46. Jagadeesh, C.H.; Ramana, R.Y.; Nagalakshmi, D.; Mahender, M.; Nalini, K.N.; Sridhar, K.; Suneetha Devi, K.B. Effect of stage of harvest on the yield, chemical composition, in-vitro and in-sacco digestibility of hybrid Napier (Pennisetum purpureum) variety APBN1. Indian J. Anim. Res. 2017, 51, 116-120. [CrossRef]

47. Lauriault, L.M.; Kirksey, R.E. Yield, and nutritive value of irrigated cereal forage grass-legume intercrops in the Southern high plains, USA. Agron. J. 2004, 96, 352-358. [CrossRef]

48. Prajapati, B.; Tiwari, S.; Kewalanand. Effect of fodder based intercropping systems on quality of fodder. Forage Res. 2018, 43, 308-313.

49. Sohail, H.K.; Abdul, G.K.; Mohammad, S.; Azim, A. Effect of maturity on production efficiency, nutritive value, and in-situ nutrients digestibility of three cereal fodders. Int. J. Agri. Res. 2007, 2, 900-909. [CrossRef]

50. Salama, H.S.A.; Zeid, M.M.K. Hay quality evaluation of summer grass and legume forage monocultures and mixtures grown under irrigated conditions. Aust. J. Crop. Sci. 2016, 10, 1543-1550. [CrossRef] 
51. Prajapati, B.; Kewalanand. Production potential of fodder based intercropping systems. Int. J. Chem. Stud. 2017, 5, 834-838.

52. Jeranyama, P.; Garcia, A.D. Understanding Relative Feed Value (RFV) and Relative Forage Quality (RFQ) 2004, Extension Extra. Paper 352. Available online: http:/ / openprairie.sdstate.edu/extension_extra/352 (accessed on 8 August 2021).

53. Karayilanli, E.; Ayhan, V. Investigation of feed value of alfalfa (Medicago sativa L.) harvested at different maturity stages. Legume Res. 2016, 39, 237-247. [CrossRef]

54. Anil, L.; Park, J.; Phipps, R.H. The potential of forage-maize intercrops in ruminant nutrition. Anim. Feed Sci. Technol. 2000, 85, 157-164. [CrossRef]

55. Prajapati, B. Cropping Systems for Sustainable Fodder and Bioenergy Production. Ph.D. Thesis, G.B. Pant University of Agriculture and Technology, Pantnagar, India, 2017.

56. Naveen, K.; Navell, C.; Sunil, K. Production efficiency and profitability of forage based cropping systems under mid hills of north-western Himalayas. Himachal J. Agric. Res. 2014, 40, 126-131.

57. Jha, S.K.; Tiwari, N. Evaluation of intensive fodder cropping systems for round the year green fodder production in Chhattisgarh. Forage Res. 2018, 44, 115-118.

58. Pandiaraj, T. Studies on Development of Climate Resilient Management Practices in Rice Based Cropping System for Higher Productivity, Resource Conservation and Soil Health. Ph.D. Thesis, G.B. Pant University Agriculture and Technology, Pantnagar, India, 2013.

59. Begna, S.; Fielding, D.; Tsegaye, T.; Van Veldhuizen, R.; Angadi, S.; Smith, D. Intercropping of oat and field pea in Alaska: An alternative approach to quality forage production and weed control. Soil Plant Sci. 2011, 61, 235-244. [CrossRef]

60. Hassen, A.; Talore, D.G.; Tesfamariam, E.H.; Friend, M.A.; Mpanza, T.D.E. Potential use of forage-legume intercropping technologies to adapt to climate-change impacts on mixed crop-livestock systems in Africa: A review. Reg. Environ. Change 2017 17, 1713-1724. [CrossRef]

61. Ates, S.; Cicek, H.; Bell, L.W.; Norman, H.C.; Mayberry, D.E.; Kassam, S.; Hannaway, D.B.; Louhaichi, M. Sustainable development of smallholder crop-livestock farming in developing countries. In IOP Conference Series: Earth and Environmental Science, Surakarta, Indonesia; IOP Publishing: Bristol, UK, 2018; Volume 142, p. 012076. [CrossRef] 